\title{
Low Reynolds number proprotor aerodynamic performance improvement using the continuous surface curvature design approach
}

\author{
Eldad J Avital $^{1^{*}}$, Theodosios Korakianitis ${ }^{2}$, Fariborz Motallebi ${ }^{1}$ \\ ${ }^{1}$ School of Engineering and Materials Science, Queen Mary University of London, London E1 4NS, \\ UK \\ ${ }^{2}$ Parks College of Engineering, Aviation and Technology, Saint Louis University, St Louis MO \\ 63103, USA
}

\begin{abstract}
Low Reynolds number blade profiles of $\mathrm{Re}_{\mathrm{C}}=10^{5}$ to $210^{5}$ as as based on chord length and used for small unnamed air vehicles, and near space applications are investigated for single and counterrotating (coaxial) proprotors, i.e. acting as rotors or propellers. Such profiles are prone for early stall, significantly reducing their maximum lift to drag ratio. Two profiles previously designed by our continuous surface curvature design approach named as CIRCLE are investigated in order to improve the performance of the proprotors. The profiles are redesigns of the common symmetric NACA0012 and asymmetric E387 profiles. Using general arguments based on composite efficiency and rotor's lift to drag ratio, the performance envelope is noticeably increased when using the redesigned profiles for high angles of attack due to stall delay.

A new approach is derived to account for the distance between the rotors of a coaxial proprotor. It is coupled with a blade element method and is verified against experimental results. Single and coaxial CIRCLE-based proprotors are investigated against the corresponding non CIRCLE-based proprotors at hover and axial translation. Noticeable improvements are observed in thrust increase and power reduction at high angles of attack of the blade's profiles, particularly for the coaxial configuration. Plots of thrust, torque, power, composite efficiency and aerodynamic efficiency distributions are given and analysed.
\end{abstract}

\section{Keywords:}

Low Reynolds number blade, Proprotor, Surface Curvature, Blade Element Method

*email: e.avital@qmul.ac.uk 


\section{List of Symbols:}
A Rotor disk area
B Number of blades
$\mathrm{C}_{\mathrm{Q}} \quad$ Rotor torque coefficient; $\mathrm{Q} /\left(\rho \mathrm{A} \Omega^{2} \mathrm{R}^{3}\right)$
$C_{P} \quad$ Rotor power coefficient; $P /\left(\rho A \Omega^{3} R^{3}\right)$
$\mathrm{C}_{\mathrm{T}}$ Rotor thrust coefficient; $\mathrm{P} /\left(\rho \mathrm{A} \Omega^{2} \mathrm{R}^{2}\right)$
C Local chord length
$\bar{c} \quad$ Geometric mean chord length
$\mathrm{C}_{\mathrm{d}}$ Profile drag coefficient
$\overline{c_{d}} \quad$ Blade averaged drag coefficient
$\mathrm{C}_{1}$ Profile lift coefficient
$\bar{C}_{l} \quad$ Blade averaged lift coefficient
$D_{e}$ Rotor's equivalent drag at forward flight
F McCormick correction function
$k_{\text {ind }}$ Ratio between the ideal and actual induced power
r Radial distance from the hub
$\mathrm{R}$ Rotor disk radius
U Free stream axial velocity
u Induced axial velocity
$\mathrm{u}_{\mathrm{m}} \quad$ Mutual induced axial velocity
$\mathrm{v}$ Induced tangential velocity
$\mathrm{V}_{\mathrm{m}} \quad$ Mutual induced tangential velocity
$\mathrm{W}_{\mathrm{e}}$ Equivalent velocity seen by the blade profile
$\mathrm{x}$ Axial distance
$\alpha$ Profile angle of attack
$\beta$ Profile pitch angle
$\beta_{0.75}$ Profile pitch angle at $r=0.75 \mathrm{R}$
$\lambda_{\infty} \quad$ Tip speed ratio; $\mathrm{U} /(\Omega \mathrm{R})$
$\mu \quad$ Advance ratio of forward flight; $\mathrm{U}_{\text {horizontal }} /(\Omega \mathrm{R})$
$\rho \quad$ Air density
$\sigma \quad$ Rotor solidity, $B \bar{c} /(\pi R)$
$\eta_{c} \quad$ Composite efficiency
$\Omega \quad$ Rotor rotational speed 


\section{Introduction}

Proprotors acting as rotors or propellers are commonly found in small unmanned aircraft (UAV). Unlike the large scale rotors or propellers, these proprotors act in a low Reynolds number flow regime of less than $\operatorname{Re}_{\mathrm{C}}$ of $310^{5}$ as based on the local chord length. Hence, the blade profiles are dominated by laminar boundary layers that are intolerant to high adverse pressure gradient, leading to early flow separation and stall as compared to the large scale blades dominated by turbulent boundary layers [1]. This makes aerodynamic approaches that delay stall of particular interest for small UAV in terms of increasing propulsion performance and hence range and endurance.

Recently the CIRCLE design method calling for continuous surface curvature and its derivative was used to redesign the NACA0012 and E387 profiles for low Re number flow [2, 3]. The method name is the acronym of presCrIbed suRface Curvature distribution bLade dEsign (CIRCLE) [4]. It is based on designing the leading and trailing edges as rounded, i.e. circles or ellipses, albeit the trailing edge is of a much smaller circle. The edges are then connected using high order polynomial and spline curves following the requirements of the profile thickness, but while keeping the surface curvature and its derivative continuous [2]. The procedure starts from the trailing edge, typically contoured as a circle with a radius of a few percentages of the profile maximum thickness and continues building the profile towards the leading edge using the high order polynomial and spline curves. Full details of the procedure are given in Shen et al [2] and Korakianitis et al [4]. It resulted in the new profiles QM13F and A7, replacing NACA0012 and E387 respectively. Redesigning the original spiky trailing edges of the NACA0012 and E387 to rounded trailing edges actually led to reduction in the production costs of the metal profiles $[3,5]$.

In case of a highly twisted and swept blade as one may encounter in turbo-machinery, the surface curvature along the blade span can also be of interest and the CIRCLE design approach has been expanded for 3D bodies as was done for subsonic and transonic compressor blades [4]. However, the typical blade for a proprotor of a small UAV is straight and moderately twisted. Hence, any discontinuity in the surface curvature will usually be at the profile plane. Therefore, this study will concentrate on adopting 2D profiles designed using the CIRCLE method for use in a proprotor.

The CIRCLE method's root for requiring continuous surface curvature in order to yield smooth pressure variation along the profile can be found in the Van Dykes’ ${ }^{\text {nd }}$ order thin aerofoil theory [2]. It was found to yield a delay in the burst of the leading edge separation bubble and thus a delay in the stall of thin aerofoil at high angle of attack (AOA). This was confirmed by RANS and Large 
Eddy Simulations (LES) carried using Ansys [2,3] and our in-house code CgLES [5]. It was also further validated using detailed wind tunnel tests [3]. The behaviour is demonstrated in Fig 1, showing the lift and drag coefficients $c_{1}$ and $c_{d}$ variations as taken from Shen et al [2, 3]. At low AOA the CIRCLE design approach was experimentally and computationally found to reduce the laminar separation bubble (LSB) over the profile's upper surface and thus mildly reduce the drag and significantly reduce the tonal trailing edge noise by up to $10 \mathrm{~dB}[3,5]$. The noise reduction occurred due a decrease in the LSB size which resulted in suppressing its interaction with the trailing edge and its flapping. In this study we will concentrate on the aerodynamic performance improvement due to the use of the CIRCLE-based blade profile in low Re number single and coaxial proprotors and leave the aeroacoustic effect for a future study.

The delay in the stall of the A7 profile as compared to the E387 profile was shown to yield about $10 \%$ increase in the power of a small wind turbine operating at a range of $\mathrm{Re}_{\mathrm{C}}=200 \mathrm{~K}$ and high $\mathrm{AOA}$ [3]. Thus it is of interest to investigate its effect on proprotors. This is not just due to the small UAV application but also due to growing interest in propeller-driven propulsion for near space vehicles as well as in extraterrestrial thin atmospheres as on Mars, where the Reynolds number is also expected to be low [6]. Asymmetric profiles as the E387 are commonly used for propellers, where symmetric profiles are more commonly used for rotors. Hence, both are investigated here for proprotor applications.

Traditionally, propeller aerodynamic performance has been analysed using the blade element method and/or models based on vortex theory [7]. This holds whether the propeller is of large scale or small scale, although the aerodynamic data for the blade profiles have to be adjusted accordingly, while also taking into account effects as compressibility and swept blades [6, 7]. The blade element method has been proved to be a mature rapid analysis tool of good accuracy [8]. More computational intensive tools as Computational Fluid Dynamics (CFD) are also available and can provide higher accuracy and more insight detail into the flow regime development around the blade [4], particularly when a turbulent flow simulation as Large Eddy Simulation (LES) is pursued [9]. However, CFD tools are much more computationally expensive than the blade element method approach.

While the blade element method whether coupled with the momentum theory or vortex theory is a mature approach to analyse a single proprotor, it is less developed for the counter-rotating (coaxial) configuration. Such configuration has been investigated since World War II and is particularly 
attractive as a rotor system to achieve balanced torque. The swirl from the front rotor is used to increase the AOA on the rear rotor blade and thus to mitigate some of the adverse effect of a higher incoming axial velocity also induced from the front rotor. The torque balance of the coaxial proprotor can be achieved by changing the rear blade pitch angle as in this study, but also by changing the rotor's rotational speed that is much easier to implement by electric motors commonly used in drones than by gas turbines used in larger aircraft. A summary of aerodynamic models used to analyse the coaxial proprotor during the decades after WWII is given in Playle et al [10].

Recently, Leswisham [11] elegantly extended the blade element momentum theory (BEMT) to the coaxial configuration assuming low AOAs and achieving good agreement with several experimental results. A simpler 1D model was developed by Beaumier [12], but both models could not explicitly account for the distance between the rotors. An attempt to account for that distance was given by Juhasz et al [13], who empirically extended the McCormick's formula of the axial velocity at the centreline induced by a helical vortex [14]. A new approach to explicitly account for the effect of the distance between the two rotors in the coaxial configuration is derived in this study using previous results of the generalized actuator disk [15]. It also accounts for large AOAs experienced by the blade profile as expected near stall.

To summarise, the aim of this study is to investigate the effect of CIRCLE-designed profiles of a continuous surface curvature on the aerodynamic performance of single and coaxial proprotors. To achieve this, the methodology is given in the next section. It is followed by analysis based on general arguments of composite efficiency and rotor's lift to drag ratio in order to assess the effect of the new profile. Aerodynamic analysis of several single and coaxial proprotors is also pursued and is followed by the conclusions section.

\section{The problem description and methodology}

This study concentrates on a single or a co-axial rotor in static or axial translation conditions. These proprotors can be viewed as helicopter rotors or aircraft propellers, depending on their design, but all can be schematically described as in Figs 2a \& 2b. Because we concentrate on low Reynolds number applications as found in small UAV, incompressibility assumption will be used. Hence straight blades will also be assumed. Extension to account for compressibility and swept back blades are possible $[6,10]$ and can be implemented in a future study. 
Traditionally, propellers have been analysed using the blade-element method approach while being coupled with the momentum method or a vortex model [7, 12]. Although, Computational Fluid Dynamics (CFD) methods are capable of providing detailed insight into the physics of the flow, they are computationally expensive as already noted. This particularly holds for the counterrotating (coaxial) rotor where an unsteady flow simulation as URANS or LES has to be implemented. Thus for this study the blade-element method has been used, which has been shown to provide accurate results for steady loads [8]. This is except for post-stall conditions where improvement in rotor power modelling is needed [16]. Nevertheless, the trend using the CIRCLEdesigned profile is still clear as will be discussed in the Results section.

The BEMT approach that was developed by Leishman [11] to deal with the co-axial proprotor relied on the assumption of small angles of attack (AOAs), while most of the improvement due to the CIRCLE design is in high AOA. Therefore the McCormick vortex model as coupled with the blade element method is used [14];

$$
\frac{4 \pi r}{B} F(r) v(r)=\frac{1}{2} c(r) c_{l}(r) W_{e}(r),
$$

where $r$ is the distance from the hub. $F$ is McCormick's correction factor that is similar to the Prandtl tip loss factor [14]. $v$ is the induced tangential velocity by the rotor on itself. $c$ is the local chord length. $c_{l}$ is the profile lift coefficient and $W_{e}$ is the equivalent velocity, see Fig $2 \mathrm{~b}$. This is an implicit equation for $v$. $F$ will be taken as of a single rotor. It is an approximation, but the analysis of Beaumier [12] and Leishman [11] have shown success by analysing each rotor as a single rotor while accounting for the effect of the other rotor just through the mutual induced velocities $u_{m} \& v_{m}$ as is done next. Furthermore, our model has shown good agreement with Leishman BEMT [11] for low AOAs and with experimental results as discussed in the next section.

Eq (1) is coupled with the following geometrical relations from Fig 2b;

$$
u+u_{m}=\frac{1}{2}\left\{-U+\left[U^{2}+4\left(v+v_{m}\right)\left(\Omega r-v+v_{m}\right)\right]\right\}^{1 / 2},
$$

and

$$
\tan (\beta-\alpha)=\frac{U-u-u_{m}}{\Omega r-v+v_{m}} .
$$

The signs of $\Omega$ and $\mathrm{v}_{\mathrm{m}}$ have been adjusted for the counter-rotating configuration. If $\mathrm{c}_{\mathrm{l}}(\alpha) \& \mathrm{c}_{\mathrm{d}}(\alpha)$ are known, then Equs (1) to (3) can be solved for $v$ using a non-linear equation solver, provided that the 
mutual induced velocities $u_{m} \& v_{m}$ are known. The solution for $v$, will also yield the value for the induced axial velocity $u$ from Eq (2).

In the BEMT model, $v_{m}=0$ on the rear rotor, while Leishman [11] took $\mathrm{u}_{\mathrm{m}}$ of the rear rotor as twice of $u$ at the front rotor, i.e. the rear rotor is the far field of the front rotor. Jushaz et al [13] used an empirical correction to McCormick's formula [14] for the axial distribution of u at the centre-line of a semi-infinite helical vortex sheet of radius $\mathrm{R}$;

$$
\frac{u(x)}{u(x=0)}=1+\left(\frac{x / R}{\sqrt{1+(x / R)^{2}}}\right)^{k} \text {. }
$$

$\mathrm{k}=1$ at the centre-line $r=0$ [14], while it decays with the radius to about 0.3 at $\mathrm{r}=\mathrm{R}$ [13].

In this study we will assume the rear rotor is in the slip stream of the front rotor and will use the actuator disk theory of Hough \& Ordway [15] that showed the steady velocity field $u(x, r)$ to be linearly dependent on an integral of the circulation contained in the disk. Assuming a uniform circulation, the distribution of $u(x, r)$ was calculated by them and is repeated in Table 1 , where $x=0$ denotes the location of the disk and $x>0$ points downstream of the disk. As seen $u(x=0, r)$ is uniform for $r<R$ and $u(x, r=0)$ follows $\mathrm{Eq}(4)$ when taking $k=1$. On the other hand the radial change in $\mathrm{u}$ is less than $20 \%$ for $r<R$ and $u$ becomes close to zero for $r>R$. The omission of any induced velocity outside the disk's stream tube was investigated by Spalart [17] to show it to be an accurate approximation, where also the change in the stream-tube from a state of climb of the rotor to decent was also investigated.

Hence, the following procedure is proposed to calculate the mutual induced velocities $u_{m} \& v_{m}$ on the rear rotor. Calculate $u \& v$ on the front rotor using the McCormick model outlined above or any blade-element method, while neglecting the effect of the rear rotor, i.e. $u_{m}=v_{m}=0$ on the front rotor. This will result in knowing $u \& v$ for $N$ radial elements of the front rotor (disk). Proceed to calculate $u_{m}$ on the rear rotor as follows;

Step 1: Take the induced axial velocity $u\left(\mathrm{x}=0, \mathrm{r}=\mathrm{r}_{\mathrm{N}}\right)$ and assume it is the same all over the front disk. Use Eq (4) to calculate the axial distribution of $u(\mathrm{x}, \mathrm{r})$ by assuming $u(\mathrm{x}, \mathrm{r})=u(\mathrm{x})$ for $\mathrm{r}<\mathrm{R}$, i.e. neglect the radial variation of $\mathrm{u}$, which we will call model 0 . Alternatively, use Table 1 to calculate $u(\mathrm{x}, \mathrm{r})$ as relative to $u\left(\mathrm{x}=0, \mathrm{r}=\mathrm{r}_{\mathrm{N}}\right)$ which we will call as model 1 . In both models we approximate $u(\mathrm{x}, \mathrm{r}>\mathrm{R})=0$. Store the value of $u(\mathrm{x}, \mathrm{r})$ at the axial location of the rear disk. 
Step 2: Subtract $u\left(\mathrm{x}=0, \mathrm{r}=\mathrm{r}_{\mathrm{N}}\right)$ from the distribution of $u(\mathrm{x}=0, \mathrm{r})$. This will result in a smaller disk of radius $\left(\mathrm{r}_{\mathrm{N}-1}+\mathrm{r}_{\mathrm{N}}\right) / 2$. Repeat Step 1 but for the new hypothetical velocity $\widetilde{u}\left(x=0, r=r_{N-1}\right)$ which is $u\left(\mathrm{x}=0, \mathrm{r}=\mathrm{r}_{\mathrm{N}-1}\right)-u\left(\mathrm{x}=0, \mathrm{r}=\mathrm{r}_{\mathrm{N}}\right)$. Add the contribution of $\mathrm{u}$ at the rear rotor to the previous value.

Step 3: Repeat Steps 2 \& 1 until all radial elements of the front disk were accounted. One should note that during this process the hypothetical velocity $\widetilde{u}(x=0, r)$ on the front disk may become negative. In that case switch between the downstream and upstream directions in Eq (4) or Table 1.

The induced tangential velocity $\mathrm{V}_{\mathrm{m}}$ can be calculated through angular momentum conservation, i.e. $v\left(\mathrm{x}=0, \mathrm{r}_{\text {front-rotor }}\right) \mathrm{r}_{\text {front-rotor }}=v\left(\mathrm{x}_{\text {rear-rotor }}, \mathrm{r}_{\text {rear-rotor }}\right) \mathrm{r}_{\text {rear-rotor. }}$. To find the relation between $\mathrm{r}_{\text {rear-rotor }}$ to $\mathrm{r}_{\text {front-rotor }}$ , the mass conservation rule is used. The induced flux $\mathrm{q}(\mathrm{r})$ on the front rotor is found by integrating $u(\mathrm{x}=0, \mathrm{r})$ and similarly $\mathrm{q}_{\mathrm{m}}(\mathrm{r})$ on the rear rotor by integrating $\mathrm{u}_{\mathrm{m}}(\mathrm{r})$ that was just found. Then the relation between $r_{\text {rear-rotor }}$ to $r_{\text {front-rotor }}$ is found by matching $q(r)$ with $q_{m}(r)$. In this step we assumed that $\mathrm{q}(\mathrm{r})$ is monotonically increasing, i.e. $\mathrm{u}(\mathrm{x}=0, \mathrm{r}) \geq 0$, so the AOA is positive. This has happened in the examples discussed in the next section. If $u(x=0, r)<0$, then the recommendation is to omit the contribution of $\mathrm{v}_{m}$ following Leishman [11].

After calculating $u_{\mathrm{m}} \& v_{\mathrm{m}}$ on the rear rotor, $u \& v$ can be calculated using the McCormick vortex detailed earlier or any other blade element method. An iterative procedure may be initiated to calculate $u_{\mathrm{m}} \& v_{\mathrm{m}}$ on the front rotor using a similar procedure as for the rear rotor. However, $v_{\mathrm{m}}$ on the front rotor is expected to be very small due to the fast decay of the swirl upstream and can be approximated as zero [12]. Our numerical experience has shown that for the kind of co-axial rotors investigated here, there is no merit in pursuing such approach. It is also supported by the BEMT approach that neglects such effect [11].

The coefficients of thrust and power of each rotor can be calculated as follows [14];

$$
\begin{aligned}
C_{T} & =\int_{0}^{1} \frac{\sigma V_{e}^{2}}{(\Omega R)^{2}}\left[c_{l} \cos (\beta-\alpha)-c_{d} \sin (\beta-\alpha)\right] d x, \\
C_{P} & =\int_{0}^{1} \frac{\sigma x V_{e}^{2}}{(\Omega R)^{2}}\left[c_{l} \cos (\beta-\alpha)+c_{d} \sin (\beta-\alpha)\right] d x .
\end{aligned}
$$

The McCormick's vortex model requires a non-linear equation solver as well as the usual requirement to balance the torque between the front and rear rotors. This can be done by varying the rear rotor rotational speed and blade pitch angle, where for the examples discussed in the next 
section it was sufficient just to vary the blade pitch angle. The robust bi-section method was used as the non-linear solver with the two loops; the inner loop for solving the McCormick's model and the outer loop to balance the torque.

Finally, a stall-delay model due to rotational augmentation was implemented following Dumirescu \& Cardos [18];

$$
c_{l}=c_{l, 2 d}+\left(c_{l, i n v}-c_{l, 2 d}\right)\left[1-e^{-1.25 /(r / c-1)}\right],
$$

where $c_{1,2 d}$ is the $c_{1}$ of the $2 \mathrm{D}$ blade profile, $c_{1, \text { inv }}$ is the $2 \mathrm{D}$ profile lift coefficient if no separation occurred, where in this study it was taken as of inviscid theory, $r$ is the radial distance from the hub and $c$ is the local chord length. Further improvement can be achieved by also correcting the profile drag coefficient $c_{d}$, but current such models usually result only in a mild improvement in the thrust prediction and no noticeable improvement in the power prediction [16].

\section{Results and analysis}

\subsection{General arguments}

Stepniewski \& Keys [19] provided two general measures to check the effect of a blade profile aerodynamics on a single rotor performance. The first measure is the figure of merit (FM) which is the ratio between the ideal power to the actual power in static (hover) condition. The second measure is the ratio between the helicopter's rotor lift to its equivalent drag during forward flight. The FM measure can be extended to axial translation of the rotor using the composite efficiency measure [11];

$$
\eta_{c}=\frac{C_{T} \lambda_{\infty}+C_{P, i d}}{C_{T} \lambda_{\infty}+C_{P, \text { ind }}+C_{P r}}
$$

where $\lambda_{\infty}=\mathrm{U} /(\Omega \mathrm{R})$ is the tip speed ratio (TSR). $\mathrm{C}_{\mathrm{P} \text {,id }}$ is the ideal induced power coefficient, i.e. uniform induced axial velocity and no tip losses. $\mathrm{C}_{\mathrm{P} \text {,ind }}$ is the actual induced power coefficient and $\mathrm{C}_{\mathrm{Pr}}$ is the profile power coefficient. In the case of a co-axial rotor, the $\mathrm{C}_{\mathrm{P}}$ 's are contributed by the

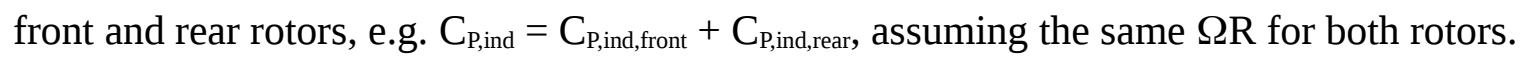

Assuming $\Omega r>>U+u$ and neglecting the effect of $v$, one can show by following the appendix that for a single rotor;

$$
\eta_{c}=\frac{3 \lambda_{\infty}+\sqrt{\lambda_{\infty}^{2}+\sigma \bar{c}_{l} / 3}}{2 \lambda_{\infty}+\left(\lambda_{\infty}+\sqrt{\lambda_{\infty}^{2}+\sigma \bar{c}_{l} / 3}\right) k_{i n d}+3{\overline{c_{d}}}_{l} /\left(2 \bar{c}_{l}\right)},
$$


where $\mathrm{k}_{\text {ind }}$ is $\mathrm{C}_{\mathrm{P} \text {,ind }} / \mathrm{C}_{\mathrm{P}, \mathrm{id}}$. $\widetilde{c_{l}}$ and $\widetilde{c_{d}}$ are the blade's averaged coefficients of lift and drag respectively and $\sigma$ is the solidity of the rotor. Eq (9) converges to the FM expression of Stepniewski \& Keys [19] when taking the tip speed ratio $\lambda_{\infty}=0$.

The variations of the composite efficiency for static conditions, rotor solidity $\sigma=0.1$ and TSR of 0.1 according to Eq (9) are given in Figs 3 \& 4 for the symmetric profiles NACA0012 \& QM13F and the asymmetric profiles E387 \& A7. QM13F \& A7 are the CIRCLE re-designed profiles and the profile aerodynamic performance was taken from Figs 1a and 1b. To account for the AOAs well after stall, post-stall empirical relations were implemented [20]. This resulted in the gradient kinks senn in the curves, particularly seen for the NACA0012 \& QM12F at AOA $\approx 15^{0}$ in Figs 3a\&b. Nevertheless, the improvement due to the re-designed profiles at high AOAs is very clear in both at static and axial translation. Increases of at least $10 \%$ in efficiency can be observed as well as flatter curves of maximum efficiency and thus providing more flexibility to the aerodynamic designer.

A general expression for the ratio of a single rotor lift $L$ to its equivalent drag $D_{e}$ at forward flight was given by Stepniewski \& Keys [19];

$$
\frac{L}{D_{e}}=\frac{1}{\left(\sigma k_{\text {ind }} \bar{c}_{l}\right) /\left(12 \mu^{2}\right)+3\left(1+4.7 \mu^{2}\right) \overline{c_{d}} /\left(4 \mu \bar{c}_{l}\right)},
$$

and is plotted in Fig 5 for all four investigated profiles and advance flight ratio $\mu=0.3$. Again the CIRCLE re-designed profiles QM13F and A7 outperform the original NACA0012 and E387 profiles at high AOAs respectively. This is caused by the better aerodynamic efficiency at high AOA. Interestingly, the asymmetric profiles show $L / D_{e}$ higher than the symmetric profiles, indicating their efficiency when it comes to moderate advance flight ratio. The rest of this section concentrates on rotors or propellers in static and axial translation conditions.

\subsection{The co-axial rotor}

The counter-rotating Harrington Rotors have been commonly used as a test case due to their detailed experimental results [21]. These are two-blades rotors, where Rotor 1 is of a tapered chord and thickness blade and Rotor 2 is of a tapered thickness blade. Both blades are untwisted and their profiles are NACA00XX where the XX stands for the profile thickness ratio.

The variations of the thrust coefficient $C_{T}$ with the torque coefficient $C_{Q}$ are plotted in Fig 6 for both rotors in static conditions, where the chord Reynolds number at $\mathrm{r}=0.75 \mathrm{R}$ was of about $1 \mathrm{M}$, and 
the profile aerodynamic data was adjusted accordingly [22]. One should note that for the co-axial rotor, the front and rear rotors were torque balanced and thus $\mathrm{C}_{\mathrm{Q}}$ is twice of the front rotor. Excellent agreement is revealed between our method and the experimental results for both the single and coaxial rotors. For the co-axial Rotor 1 , not much difference is revealed between Model 0 and Model 1 that differ in calculating the radial variation of $\mathrm{u}_{\mathrm{m}}$ along the rear rotor. However, for co-axial Rotor 2, Model 1 that better accounts for the radial variation of $u_{m}$ outperforms Model 0 .

To examine the effect of replacing NACA0012 with QM13F, Rotor 2 was rescaled for the Reynolds number of $\mathrm{Re}_{\mathrm{C}}=1.3510^{5}$ as in Fig 1a and modified to have a uniform profile of NACA 0012 or QM13F. This means the blade is uniform in chord length, thickness and $c_{l} \& c_{d}$ variations with the AOA. It is an approximation of a hypothetical rotor that aims to investigate the difference between the two profiles and not to provide exact results for a particular rotor. The variation of the figure of merit with the overall thrust coefficient is shown in Fig 7 for the single and co-axial rotor configurations. The QM13F based rotors show improved FM at high $\mathrm{C}_{\mathrm{T}}$ due to the improved aerodynamic efficiency of QM13F at high AOA. The improvement is more noticeable in the coaxial rotor than in the single rotor.

To better understand the effect of changing the NACA0012 profile with the QM13F profile, contours of the aerodynamic efficiency $c_{l} / c_{d}$ are plotted in Fig 8 as a function of the radial location and the blade pitch angle of the front rotor. As the mutual induced velocities $u_{\mathrm{m}} \& v_{\mathrm{m}}$ are neglected in the front rotor calculation, the contours for the front rotor should also be viewed as for the single rotor.

It is seen that the front rotor's regime of reduced aerodynamic efficiency at high pitch angle is mildly reduced for the QM13F profile as compared to the NACA0012 profile, thus resulting in the moderate improvement in the FM of the single rotor seen in Fig 7. The rear rotor shows two opposite patterns of behaviour. The area of $r>(0.7 \div 0.8) R$ that is outside of the wake shed by the front rotor, shows a behaviour similar to that of the front rotor, i.e. increase in the aerodynamic efficiency with the pitch angle until stall has reached and then a decrease in the aerodynamic efficiency due to post stall condition. The area that is inside the front rotor wake of $\mathrm{r}<(0.7 \div 0.8) R$ shows low aerodynamic efficiency at low pitch angles because of the high $\mathrm{u}_{\mathrm{m}}$ caused by the front rotor which reduces the AOA on the rear rotor. However, at high pitch angle the front rotor stalls and $u_{\mathrm{m}}$ is much reduced causing the AOA to increase on the rear rotor for $\mathrm{r}<(0.7 \div 0.8) R$ as seen in Figs 8b\&8d. The sharp border in Figs 8c and 8d between the area affected by the front rotor wake 
and the area that does not, is obviously an approximation of the wake effect, similar to the approach used in Lesisham's [11] proprotor model for when the rear rotor was in the far field of the front rotor's wake. Nevertheless, the model yields accurate results as seen in Fig 6. On overall, the coaxial rotor based on the QM13F profile outperforms the NACA0012-based coaxial rotor in high pitch angles, i.e. high thrust coefficient as seen Fig 7.

The variation of the composite efficiency with the tip speed ratio (TSR) for the small rescaled rotor is shown in Fig 9 for both the single and coaxial configurations. As the TSR increases the AOA seen by the blade profile decreases and thus the difference between the QM13F and NACA0012-based blades diminishes. Hence, the highest difference is at static condition for a fixed pitch angle, where the composite efficiency becomes the figure of merit of Fig 7. Interestingly, the composite efficiency initially increases for the coaxial rotor and high pitch angle of $18^{0}$ as the TSR is increased from zero. This is related to the behaviour of the rear rotor as seen in Fig 8.

\subsection{The single low Reynolds number propeller.}

Symmetric profiles as the NACA0012 are commonly used for rotors, while asymmetric profiles are more commonly used for propellers. Experimental measurements were provided by Ghoddoussi [23] and his small scale two blade COMP propeller based on the E387 was picked up for this study. The variations of the thrust and power coefficients with the TSR are given in Fig 10, where for the model, the $\mathrm{C}_{\mathrm{l}} \& \mathrm{c}_{\mathrm{d}}$ variations were based on $\mathrm{Re}_{\mathrm{C}}=10^{5}$. For consistency the experimental propeller results were re-adjusted for the definitions of the thrust and power coefficients, and tip speed ratio as commonly used for rotor aerodynamics and as are used in this study.

Very good agreement is revealed between the experimental and model results for high TSR or low blade pitch angle, where both the E387 and A7 propellers give the same result. This is because of the low AOAs seen by the propeller blades. At high pitch angles and low TSR big differences can be seen between the experimental and model results, particularly for $C_{p}$. This is because of the current inadequacy of stall-delay models to accurately predict the power [16], pointing to the need to improve such models. Nevertheless, the trend caused by replacing E387 by the redesigned A7 is consistent and clear. It results in a higher $\mathrm{C}_{\mathrm{T}}$ and a lower $\mathrm{C}_{\mathrm{P}}$ for low TSRs and high pitch angles. This is as expected from the better aerodynamic efficiency of the A7 at high AOA, pointing to the benefit in using the CIRCLE-based profile,

\section{Summary}


Two low Reynolds number redesigned blade profiles QM13F and A7 were investigated for their effect on thrust and power of single and coaxial proprotors. The QM13F is a redesign of the NACA0012 profile and the A7 is a redesign of the E387 profile. Both redesigns followed the CIRCLE procedure calling for a continuous surface curvature and its derivative along the profile. Previously published results of $c_{l} \& c_{d}$ verses AOA of both profiles for $\operatorname{Re}_{\mathrm{C}}<310^{5}$ were used and coupled with a blade-element method based on the McCormick vortex model. To model the coaxial rotor, the rear rotor was assumed to be in the slipstream of the front rotor. A new method as based on the generalised actuator disk theory was proposed to account for the effect of the distance between the rotors, leading to a formulated approach to calculate the mutual induced velocities acting on the rear rotor. For the investigated coaxial rotor configurations, it was found to be sufficient to neglect the mutual induced velocity on the front rotor.

General arguments were used to produce analysis based on the composite efficiency measure at axial translation and rotor's lift to equivalent drag at forward flight. This was followed by blade element method analysis of several single and coaxial proprotors, leading to the following conclusions;

- The blade-element method coupled with the McCormick method and the new approach to account for the distance between the rotors of a coaxial configuration, produced excellent agreement with experimental results in terms of thrust and torque/power. However, at highly stalled blades the need for imported stall-delay models was highlighted particularly for the power prediction.

- The higher aerodynamic efficiency of the CIRCLE-based blade at high AOA leads to a wider envelope of high efficiency and more flexibility for the aerodynamic designer.

- The performance improvement was more noticeable in the coaxial configuration than the single configuration due to the behaviour the rear rotor blade. The blade produced high aerodynamic efficiency at the slip stream region of the front rotor after the latter stalled. This is because of a reduction in $\mathrm{u}_{\mathrm{m}}$ acting on the rear rotor.

Straight and moderately twisted blades were considered in this study as one may expect for proprotors of small UAVs. However, as already noted when highly twisted and swept blades are used as in propfans, discontinuities in the surface curvature may occur along the blade's span and the CIRCLE approach should be applied three-dimensionally as it was in turbo-machinery applications [4]. This study concentrated on the aerodynamics of the proprotor, but as noted earlier the CIRCLE-based profile can yield a reduced tonal self noise at the low Reynolds numbers studied here. To what degree it affects the overall noise generation by the proprotor that includes other 
components of noise as the dominant thrust-torque noise and wake noise is an open research question. 


\section{References:}

[1] Mueller TJ and DeLaurier JD, Aerodynamics of small vehicles, Annu Rev Fluid Mech 35, 2003, 89-111

[2] Shen X, Avital E, Rezaienia MA, Paul G, Korakianitis T, Computational methods for investigation of surface curvature effects on airfoil boundary layer behavior, J Algorithms \&Compl Tech 11(1), 2017, 68-82

[3] Shen X, Avital E, Paul G, Rezaienia MA, Wen P, Korakianitis T, Experimental study of surface curvature effects on aerodynamics of low Reynolds number airfoil for small wind turbines $J$ Ren Sustain Energy 8 (5), 2017, 053303

[4] Korakianitis T, Hamakham IA, Rezaienia MA, Wheeler APS, Avital EJ and Williams JJR, Design of high-efficiency turbomachinery blades for energy conversion devices with threedimensional prescribed surface curvature distribution blade design (CIRCLE) method, Applied Energy 89, 2012, 215-227

[5] Shen X, Avital E, Zhao Q, Gao J, Li X, Paul G, Korakianitis T, Surface curvature effects on the tonal noise performance of a low Reynolds number aerofoil, Applied Acous 125, 2017, 34-40

[6] Zhang S, Yang X, Song B and Song W, Aerodynamic design of a novel low-Reynolds-number airfoil for near space propellers, Proc 2015 Asian-Pacific Conf Aero Tech Sci, Jeju Korea, 603-607

[7] Wald Q, The aerodynamics of propellers, Prog Aero Sci 42, 2006, 85-128

[8] Gur O and Rosen A, Comparison between blade-element models of propellers, Aero $J$ 112(1138), 2008, 689-704

[9] Bai X, Avital EJ, Munjiza A and Williams JJR, Numerical Simulation of a marine current turbine in free surface flow. Renewable Energy 63, 2014, 715-723

[10] Playle SC, Korkan KD and Lavante, A Numerical method for the design and analysis of counter-rotating propellers, AIAA J Propulsion 2(1), 1986, 57-63

[11] Leishman JD, Aerodynamic performance considerations in the design of a coaxial proprotor, $J$ Am Helic Soc 54, 2009, 012005

[12] Beaumier P, A low-order method for co-axial propeller and rotor performance prediction, ICAS 2014 St Petersbourg, hal-0107955

[13] Juhasz O, Syal M, Celi R, Khromov V, Rand O, Ruzicka GC and Strawn RC, Comparison of three coaxial aerodynamic prediction methods including validation with model test data, J Am Helic Soc 59, 20014, 032006

[14] McCormick BW, Aerodynamics of V/STOL flight, 1999, Dover Publications, Mineola N-Y, 7992, 96-97

[15] Hough GR and Ordway DE, The generalized actuator disk, 1964, TAR-TR 6401 
[16] Morgado J, Silvestre AR and Pascoa, A Comparison of post-stall models extended for propeller performance prediction, Aircraft Eng Aero Tech: Int J 88(4), 2016, 540-549

[17] Spalart PR, On the simple actuator disk, J Fluid Mech 494, 2003, 399-405

[18] Dumitrescu H and Cardos V, A stall-delay model for rotating blades, Proc Appl Math Meth 7, 2007, 4100003

[19] Stepniewski WZ and Keys CN, Rotary-wing aerodynamics, 1984, Dover Pub, New-York, 350355

[20] Tangler \& Kocurek, Wind turbine post-stall airfoil performance characteristics guidelines for BEM methods, 2004, NREL/CP-500-36900

[21] Harrington RD, Full-scale-tunnel investigation of the static-thrust performance of a coaxial helicopter rotor, 1951, NACA TN2318

[22] Sheldahl RE and Kilmas PC, Aerodynamic characteristics of seven symmetrical airfoil sections through 180-degree angle for use in aerodynamic analysis of vertical axis wind turbines, Sandia National Laboratories, 1981, SAND80-2114

[23] Ghoddoussi A, A More comprehensive database for propeller performance validations at low Reynolds numbers, $\mathrm{PhD}$ thesis, Wichita State University, 2016

\section{Appendix - the composite efficiency, general approximation}

The thrust coefficient $\mathrm{C}_{\mathrm{T}}$ and the ideal power coefficient $\mathrm{C}_{\mathrm{p} \text {,id }}$ of a single rotor can be taken as;

$$
C_{T}=2 \lambda_{i d}\left(\lambda_{\infty}+\lambda_{i d}\right), C_{P, i d}=C_{T}\left(\lambda_{\infty}+\lambda_{i d}\right),
$$

where $\lambda_{\mathrm{id}}=\mathrm{u}_{\mathrm{id}} /(\Omega \mathrm{R})$ and $\lambda_{\infty}=\mathrm{U} /(\Omega \mathrm{R})$ [19]. $\mathrm{u}_{\mathrm{id}}$ is the ideal uniform induced axial velocity. The ratio between the axial induced power and the ideal power is defined as $k_{\text {ind }}=\mathrm{C}_{\mathrm{p} \text {,ind }} / \mathrm{C}_{\mathrm{P} \text {,id. }}$. Assuming $\Omega r>>(U+u)$ and thus low flow angle $\beta-\alpha$, one gets that;

$$
C_{P r}=\sigma \bar{c}_{d} / 8, C_{T}=\sigma \bar{c}_{l} / 6,
$$

Substituting Eqs (A1) \& (A2) into the expression of the composite efficiency in Eq (8) leads to the general expression of Eq (9), after expressing $\lambda_{\text {id }}$ as a function of $\lambda_{\infty}$ and the blade's averaged lift coefficient $\bar{c}_{l}$. 


\begin{tabular}{|l|l|l|l|l|l|l|l|l|l|}
\hline & -2.0 & -1.0 & -0.5 & -0.1 & $x / R=0$ & 0.1 & 0.5 & 1.0 & 2.0 \\
\hline$r / R=0$ & 0.026 & 0.073 & 0.138 & 0.225 & 0.250 & 0.275 & 0.362 & 0.427 & 0.474 \\
\hline 0.1 & 0.026 & 0.073 & 0.138 & 0.225 & 0.250 & 0.275 & 0.362 & 0.427 & 0.474 \\
\hline 0.2 & 0.026 & 0.072 & 0.136 & 0.224 & 0.250 & 0.276 & 0.364 & 0.428 & 0.474 \\
\hline 0.3 & 0.026 & 0.070 & 0.133 & 0.223 & 0.250 & 0.277 & 0.367 & 0.430 & 0.474 \\
\hline 0.4 & 0.025 & 0.068 & 0.129 & 0.222 & 0.250 & 0.278 & 0.371 & 0.432 & 0.475 \\
\hline 0.5 & 0.025 & 0.065 & 0.124 & 0.219 & 0.250 & 0.281 & 0.377 & 0.435 & 0.475 \\
\hline 0.6 & 0.024 & 0.062 & 0.116 & 0.215 & 0.250 & 0.285 & 0.384 & 0.438 & 0.476 \\
\hline 0.7 & 0.023 & 0.058 & 0.107 & 0.209 & 0.250 & 0.291 & 0.393 & 0.442 & 0.477 \\
\hline 0.8 & 0.022 & 0.054 & 0.096 & 0.197 & 0.250 & 0.303 & 0.404 & 0.446 & 0.478 \\
\hline 0.9 & 0.022 & 0.049 & 0.084 & 0.170 & 0.250 & 0.330 & 0.416 & 0.451 & 0.478 \\
\hline 1.0 & 0.021 & 0.045 & 0.070 & 0.108 & 0.125 & 0.142 & 0.180 & 0.205 & 0.229 \\
\hline 1.1 & 0.020 & 0.040 & 0.058 & 0.047 & 0 & -0.047 & -0.058 & -0.040 & -0.020 \\
\hline 1.2 & 0.019 & 0.036 & 0.046 & 0.024 & 0 & -0.024 & -0.046 & -0.036 & -0.019 \\
\hline 1.5 & 0.016 & 0.025 & 0.024 & 0.007 & 0 & -0.007 & -0.024 & -0.025 & -0.016 \\
\hline 2.0 & 0.011 & 0.013 & 0.009 & 0.002 & 0 & -0.002 & -0.009 & -0.013 & -0.011 \\
\hline 3.0 & 0.005 & 0.004 & 0.003 & 0.001 & 0 & -0.001 & -0.003 & -0.004 & -0.005 \\
\hline 5.0 & 0.002 & 0.001 & 0.001 & 0 & 0 & 0 & -0.001 & -0.001 & -0.002 \\
\hline
\end{tabular}

Table 1: Normalized axial velocity $u /\left(U C_{T}\right)$ distribution as induced by an actuator disk of blade constant circulation [15]. 


\section{List of Figures:}

Figure 1: The lift and drag coefficients variations with the angle of attack that are plotted for the profiles (a) NACA0012 and the CIRCLE-redesigned QM13F of $\operatorname{Re}_{\mathrm{D}}=1.3510^{5}$ [2] and (b) E387 and the CIRCLE-redesigned A7 of $\mathrm{Re}_{\mathrm{D}}=2105$ [3].

Figure 2: Schematic description of the co-axial proprotor

Figure 3: The figure of merit variations with the profile angle of attack that are plotted for generic single rotor disks having the profiles (a) NACA0012 or QM13F and (b) E387 or A7 of Fig 2. $k_{\text {ind }}$ the ratio of actual to induced ideal power equally varies from 1.1 to 1.5

Figure 4: The composite efficiency variations with the profile angle of attack that are plotted for the generic rotor disks of Fig. 3 at axial translation with a tip speed ratio of $\lambda_{\infty}=0.1$.

Figure 5: The rotor's lift to equivalent drag ratio variations with the profile angle of attack that are plotted for the generic rotor disks of Fig. 3 and at horizontal flight with an advance speed ratio $\mu=0.3$

Figure 6: Variations of the thrust and torque coefficients of the Harrington two-blade rotors' staticthrust tests [21] and which are plotted for (a) Rotor 1 of the tapered chord and thickness blade and (b) Rotor 2 of the tapered thickness blade. Model 0 means $\mathrm{u}(\mathrm{x}, \mathrm{r})=\mathrm{u}(\mathrm{x}, \mathrm{r}=0)$ of Table 1 for $\mathrm{r}<\mathrm{R}$. Model 1 means $\mathrm{u}(\mathrm{x}, \mathrm{r})$ is found using Table 1 .

Figure 7: The variation of the figure of merit with the static thrust coefficient for a small re-scaled Harrington Rotor 2, where the blade profile is uniformly NACA0012 or QM13F of Fig 1a.

Figure 8: The spanwise variation of the blade profile's lift to drag ratio for the range of untwisted blade pitch angle $\beta$ corresponding to the FM plots of Fig 7 and which are plotted for (a) the upper rotor with the NACA0012 profile, (b) the upper rotor with the QM13F profile, (c) the lower rotor with the NACA0012 profile and (d) the lower rotor with the QM13F.

Figure 9: Variation of the composite efficiency $\eta_{c}$ with the tip speed ratio $\lambda_{\infty}$ for the small rescaled (a) single and (b) coaxial Harrington Rotor 2, where both are at axial translation and $\beta$ is the untwisted blade pitch angle.

Figure 10: The variations of the (a) thrust and (b) power coefficients for the two-blade twisted COMP propeller having the E387 profile [23] and the redesigned A7 profile. 
(a)

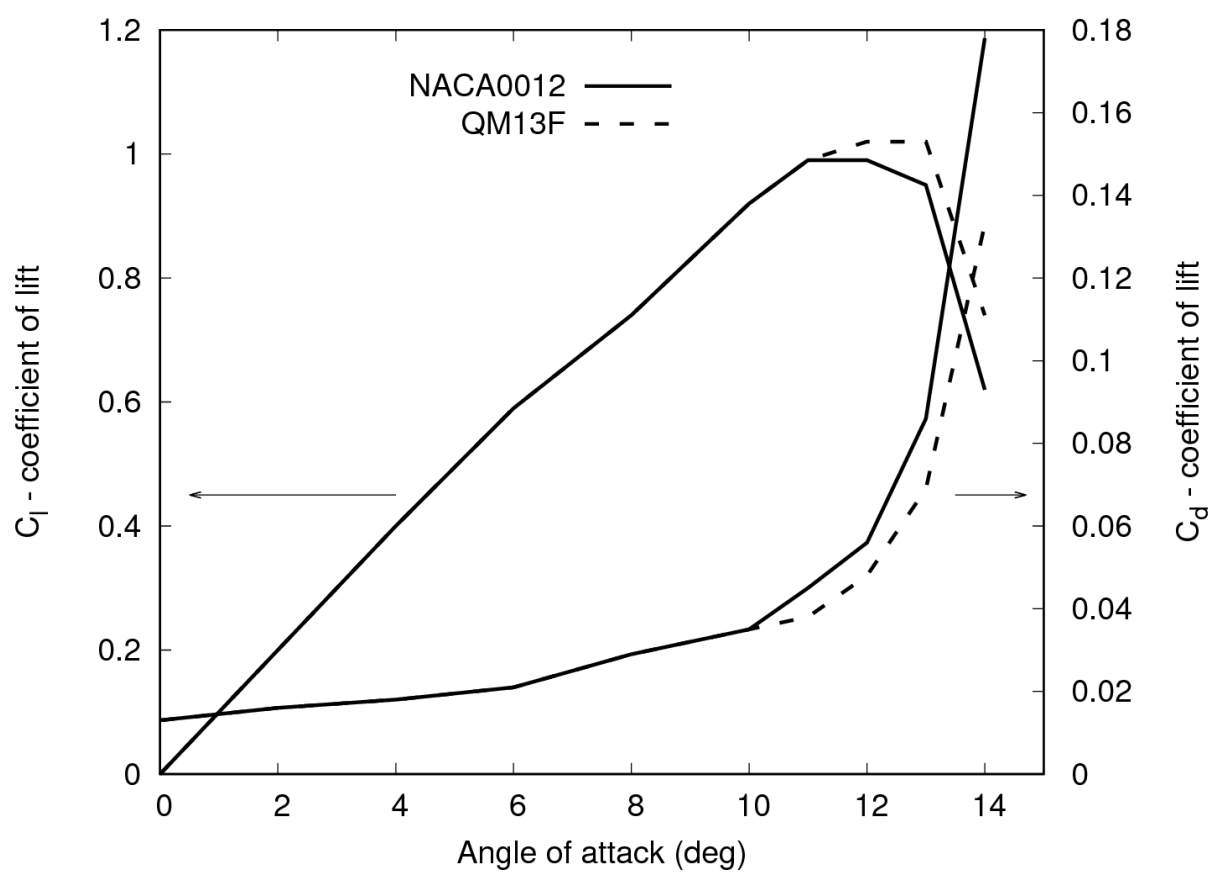

(b)

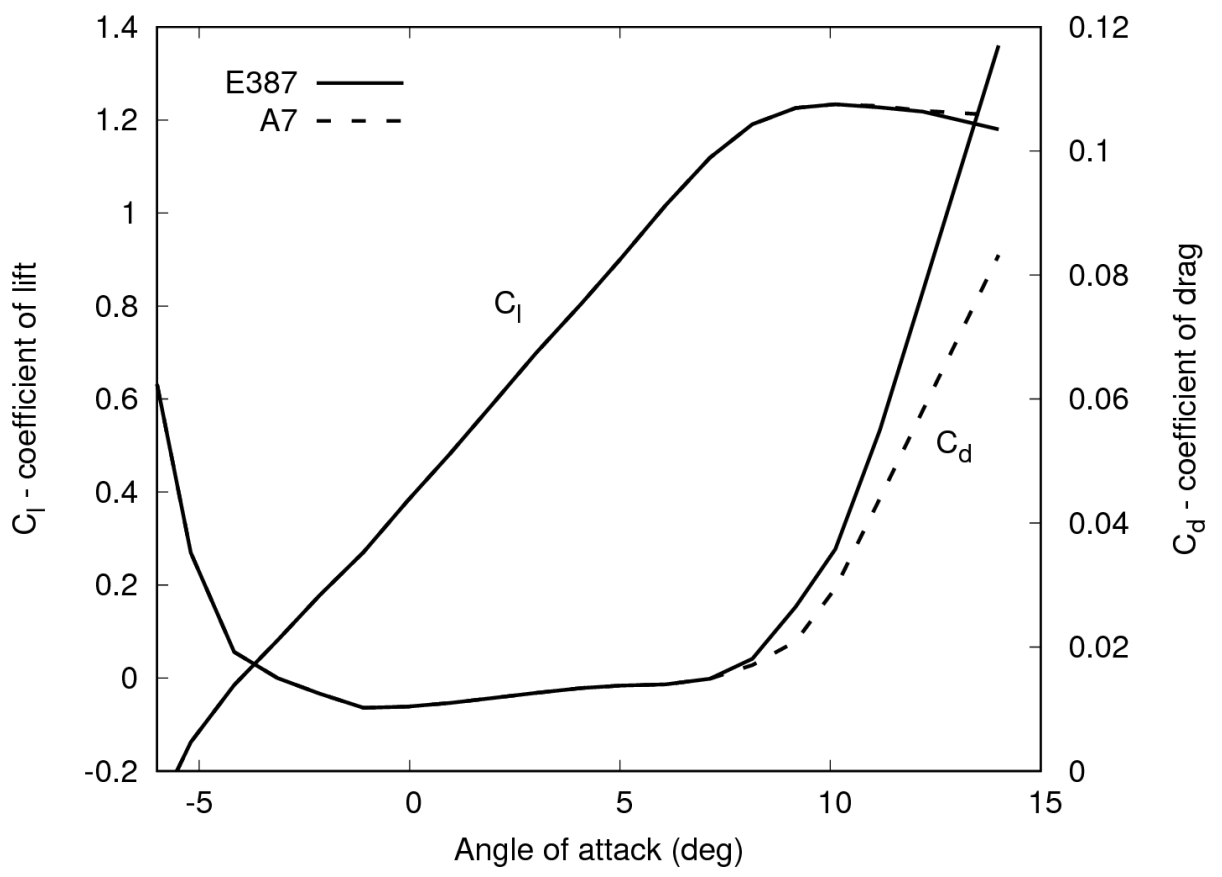

Figure 1: The lift and drag coefficients variations with the angle of attack that are plotted for the profiles (a) NACA0012 and the CIRCLE-redesigned QM13F of $\operatorname{Re}_{\mathrm{D}}=1.3510^{5}$ [2] and (b) E387 and the CIRCLE-redesigned A7 of $\mathrm{Re}_{\mathrm{D}}=2105$ [3]. 
(a)

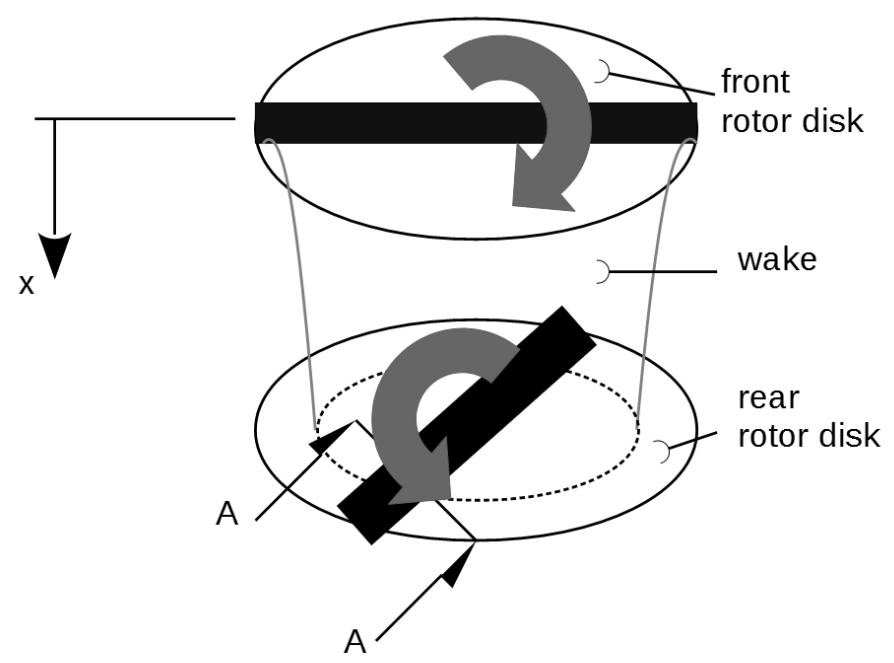

(b)

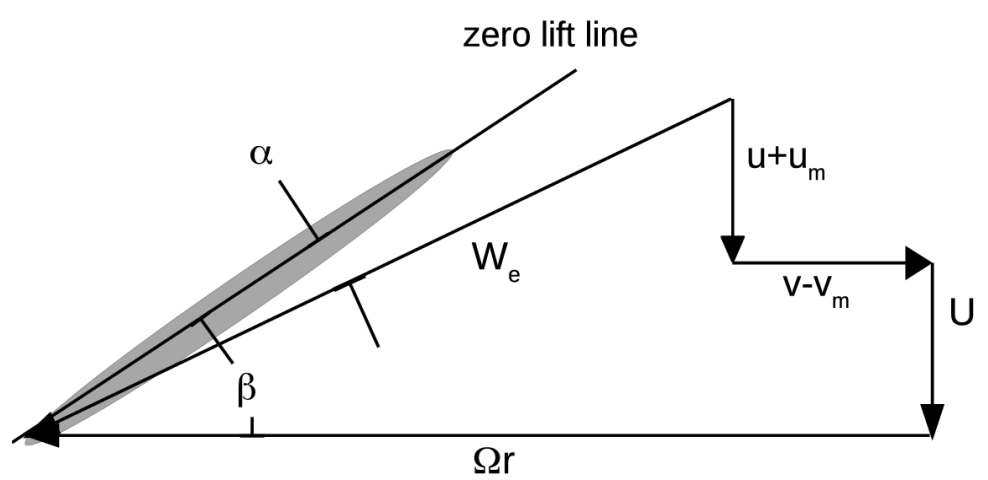

Section A-A

Figure 2: Schematic description of the co-axial proprotor 
(a)

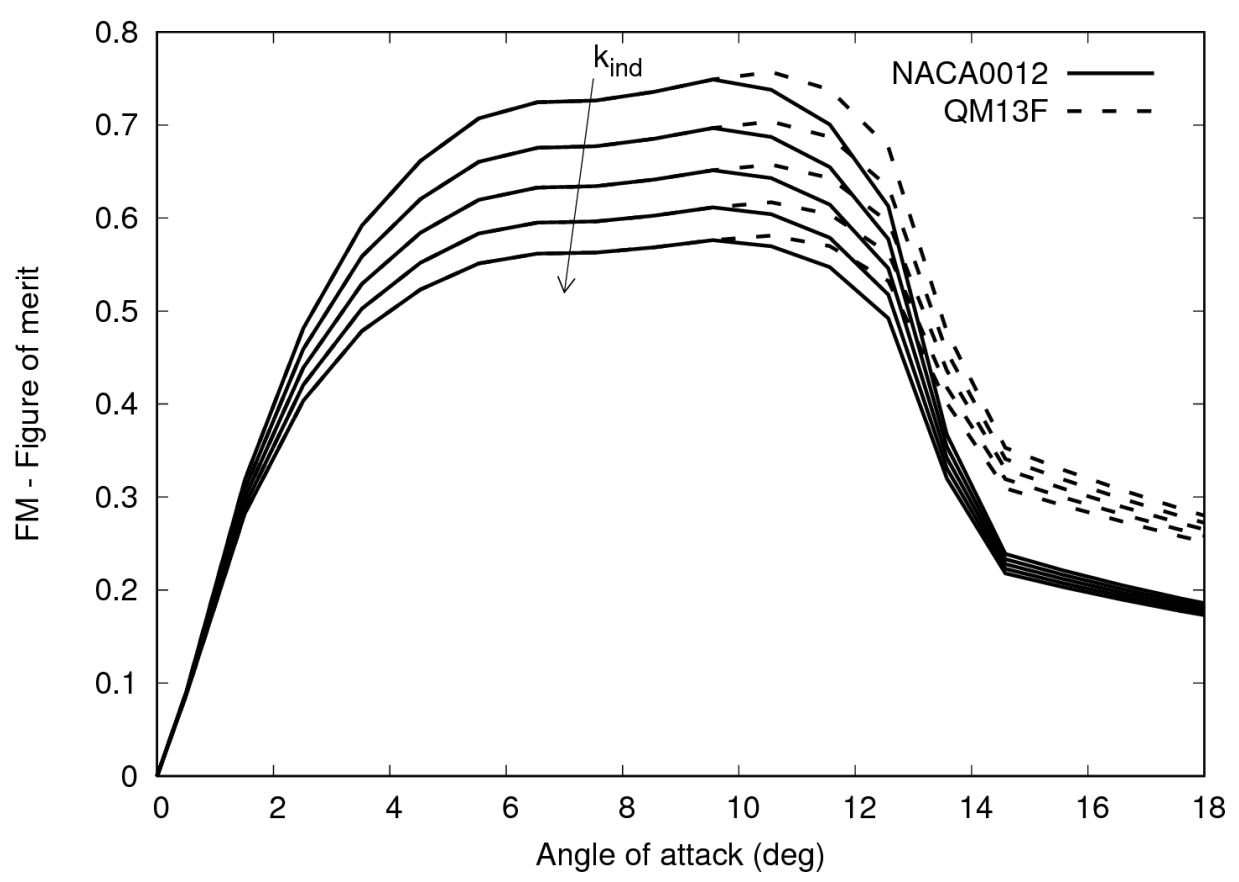

(b)

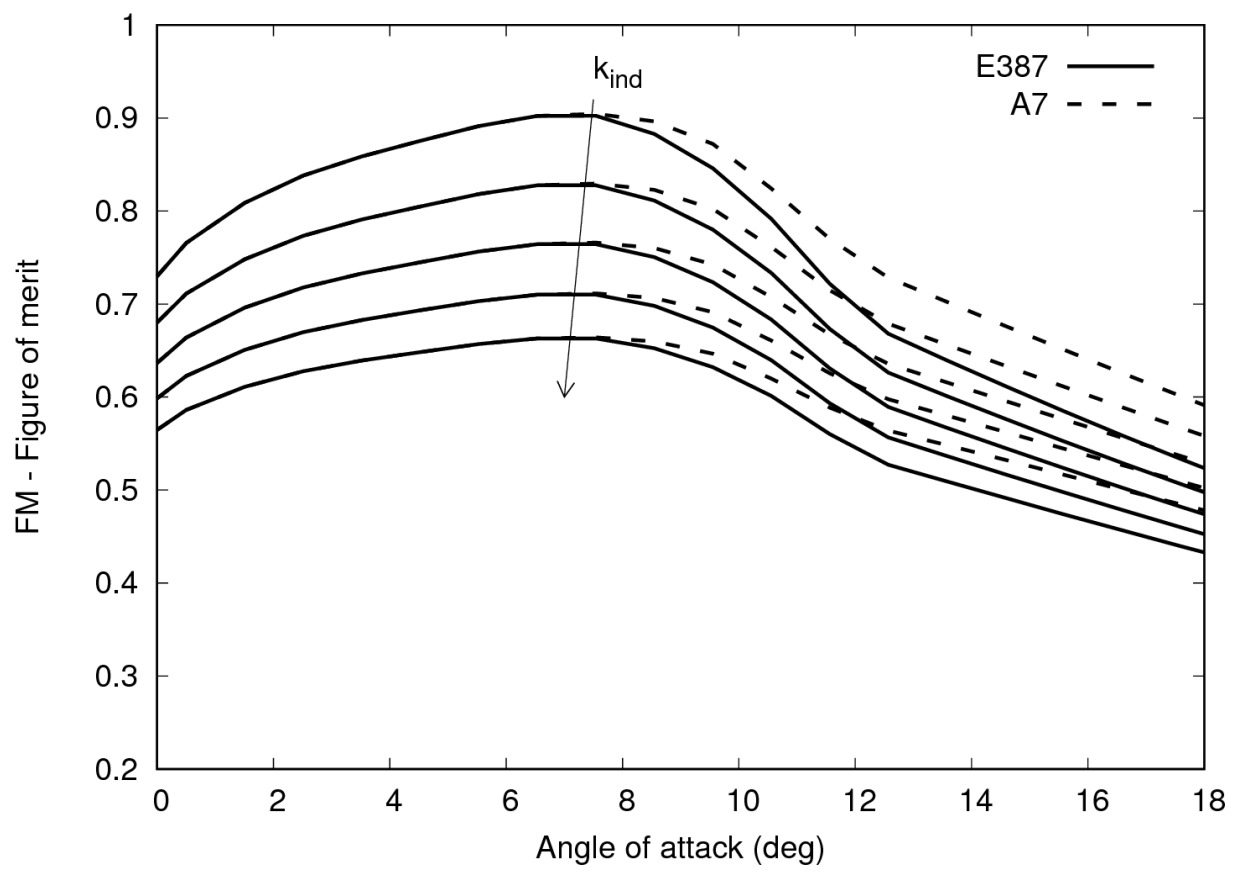

Figure 3: The figure of merit variations with the profile angle of attack that are plotted for generic single rotor disks having the profiles (a) NACA0012 or QM13F and (b) E387 or A7 of Fig 2. $k_{\text {ind }}$ the ratio of actual to induced ideal power equally varies from 1.1 to 1.5 
(a)

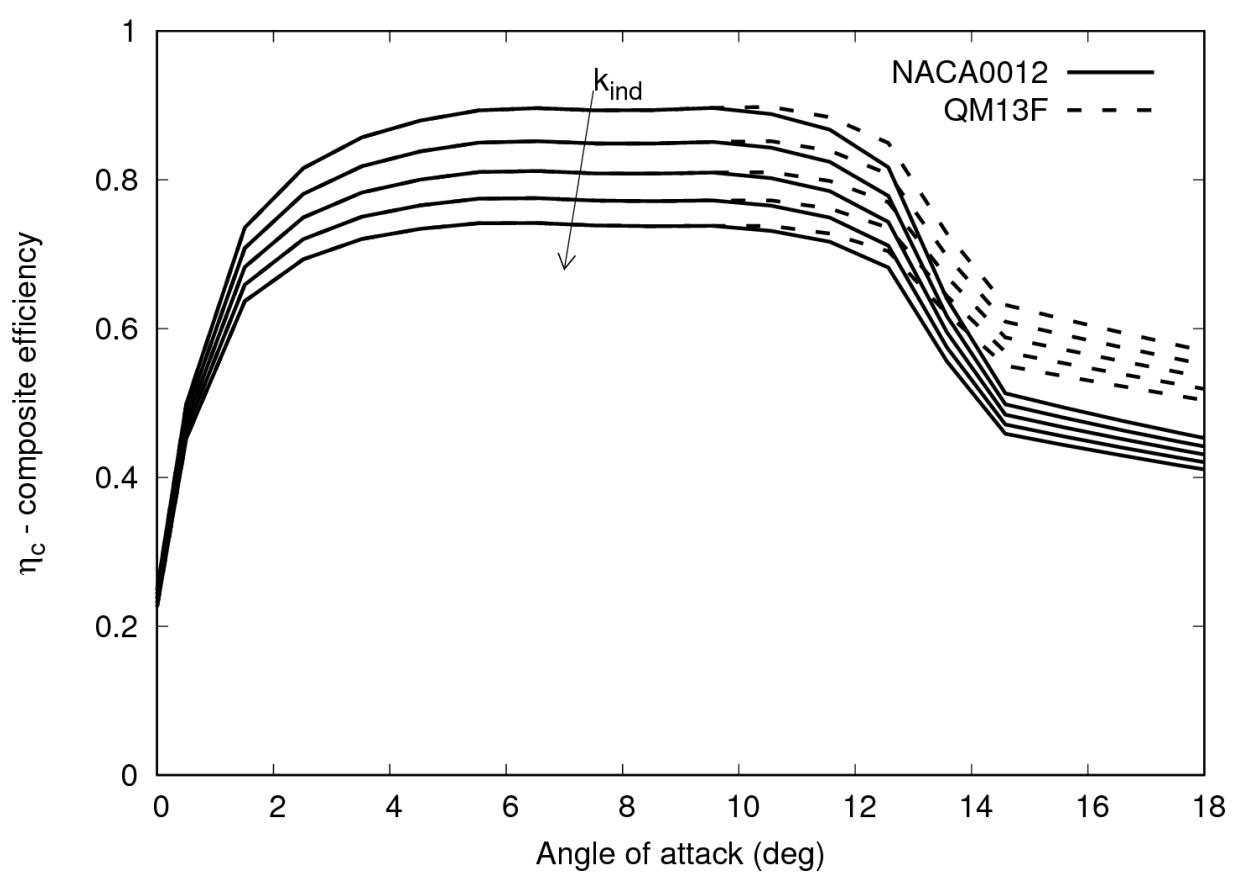

(b)

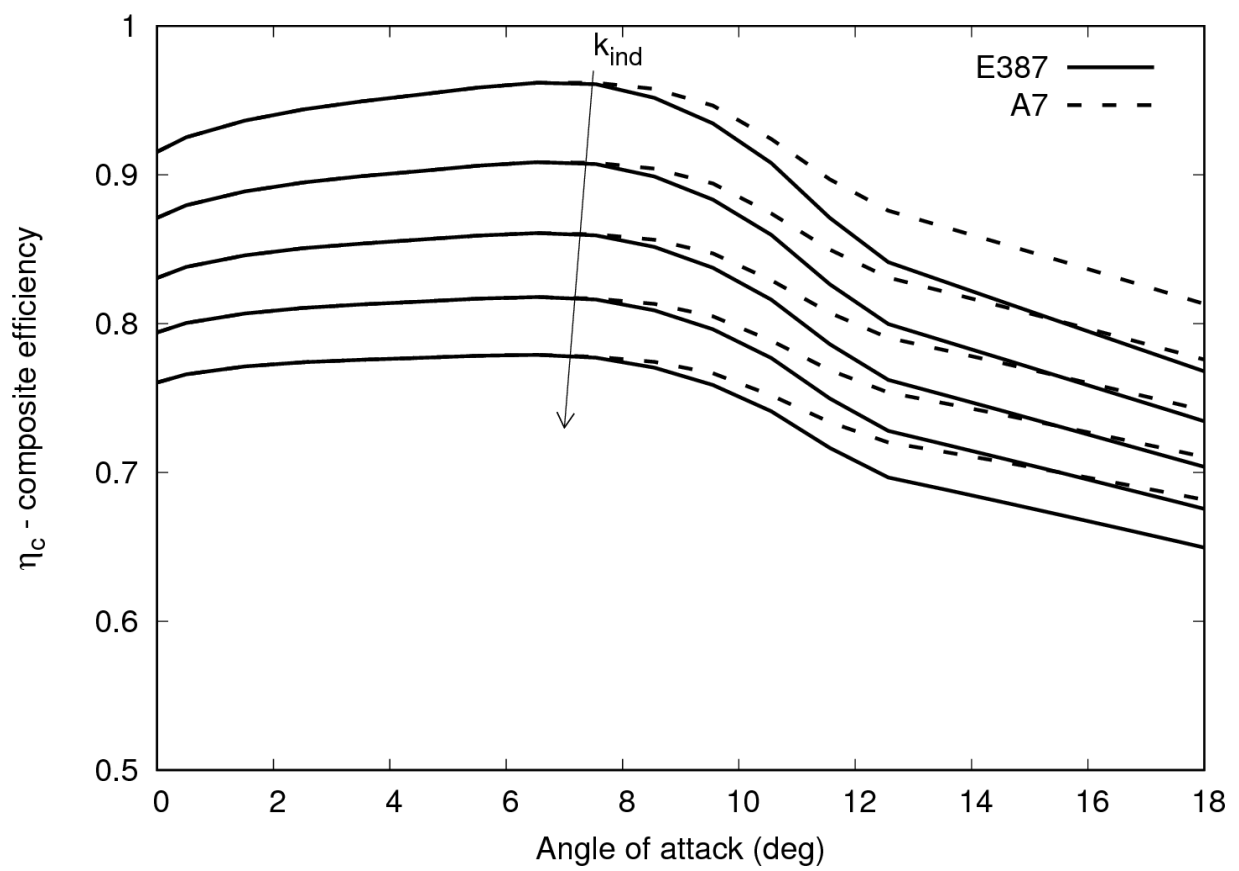

Figure 4: The composite efficiency variations with the profile angle of attack that are plotted for the generic rotor disks of Fig. 3 at axial translation with a tip speed ratio of $\lambda_{\infty}=0.1$. 
(a)

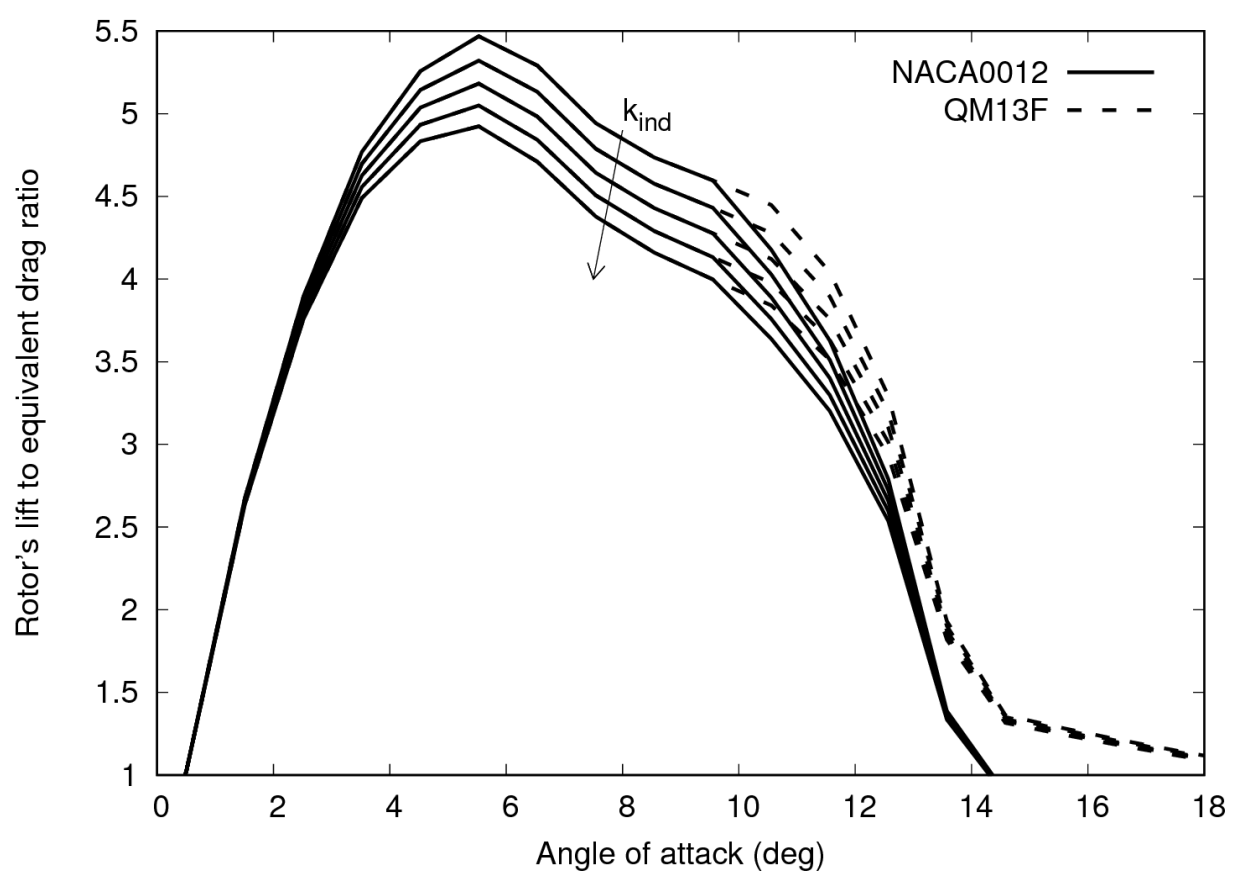

(b)

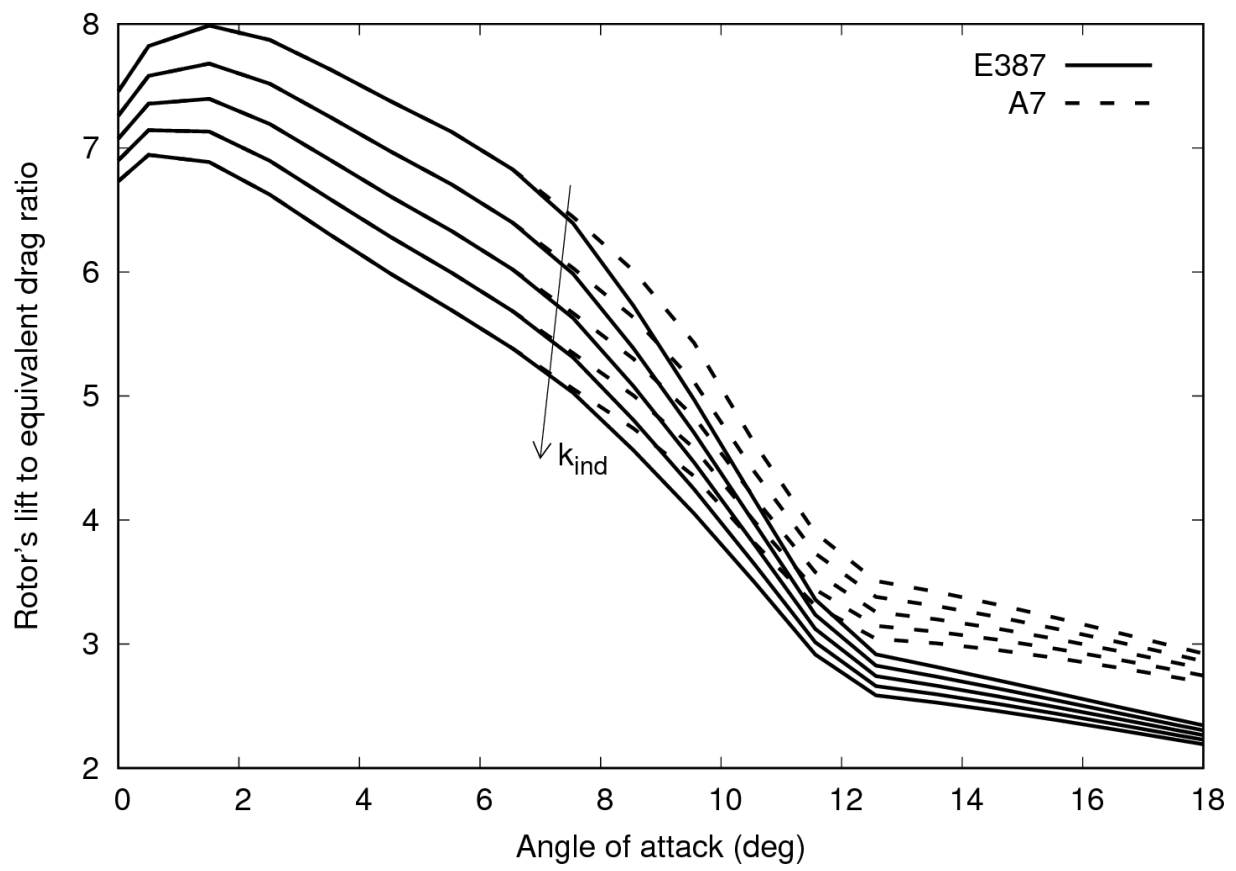

Figure 5: The rotor's lift to equivalent drag ratio variations with the profile angle of attack that are plotted for the generic rotor disks of Fig. 3 and at horizontal flight with an advance speed ratio $\mu=0.3$ 
(a)

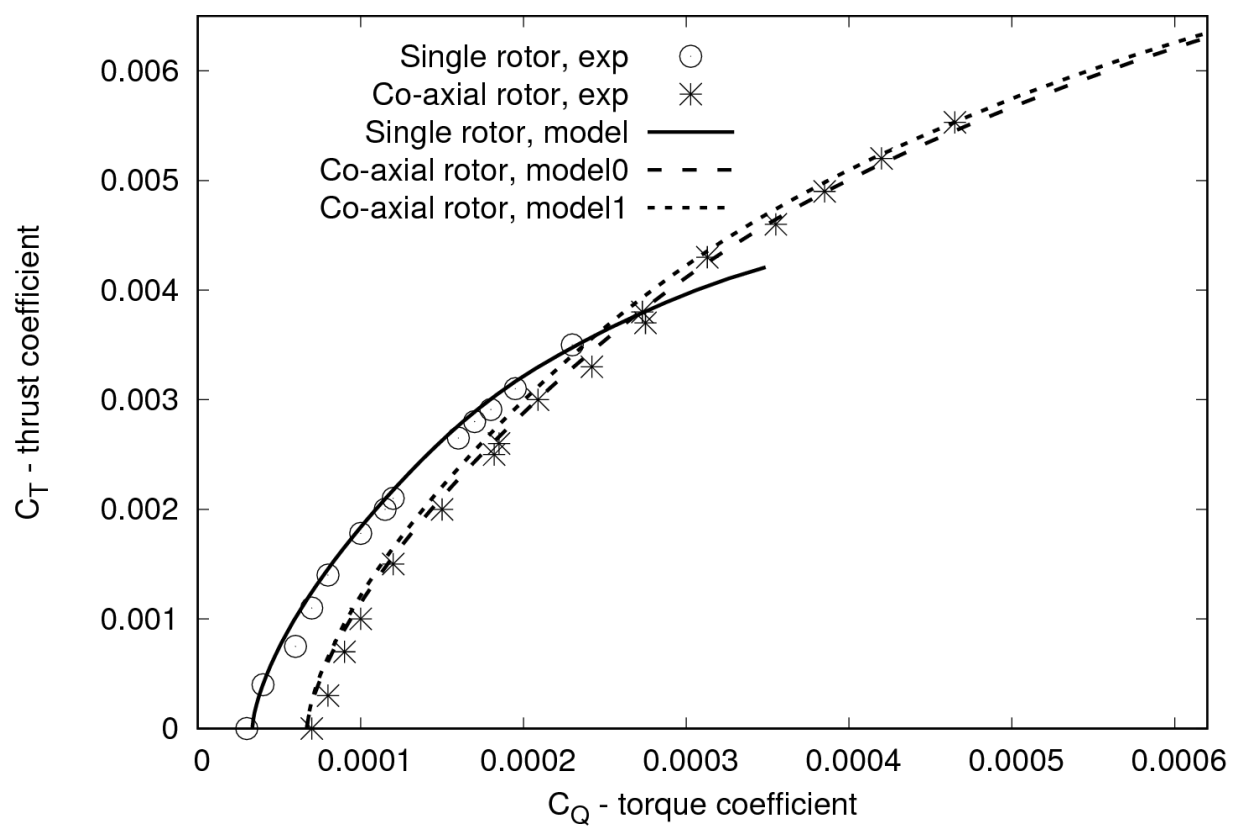

(b)

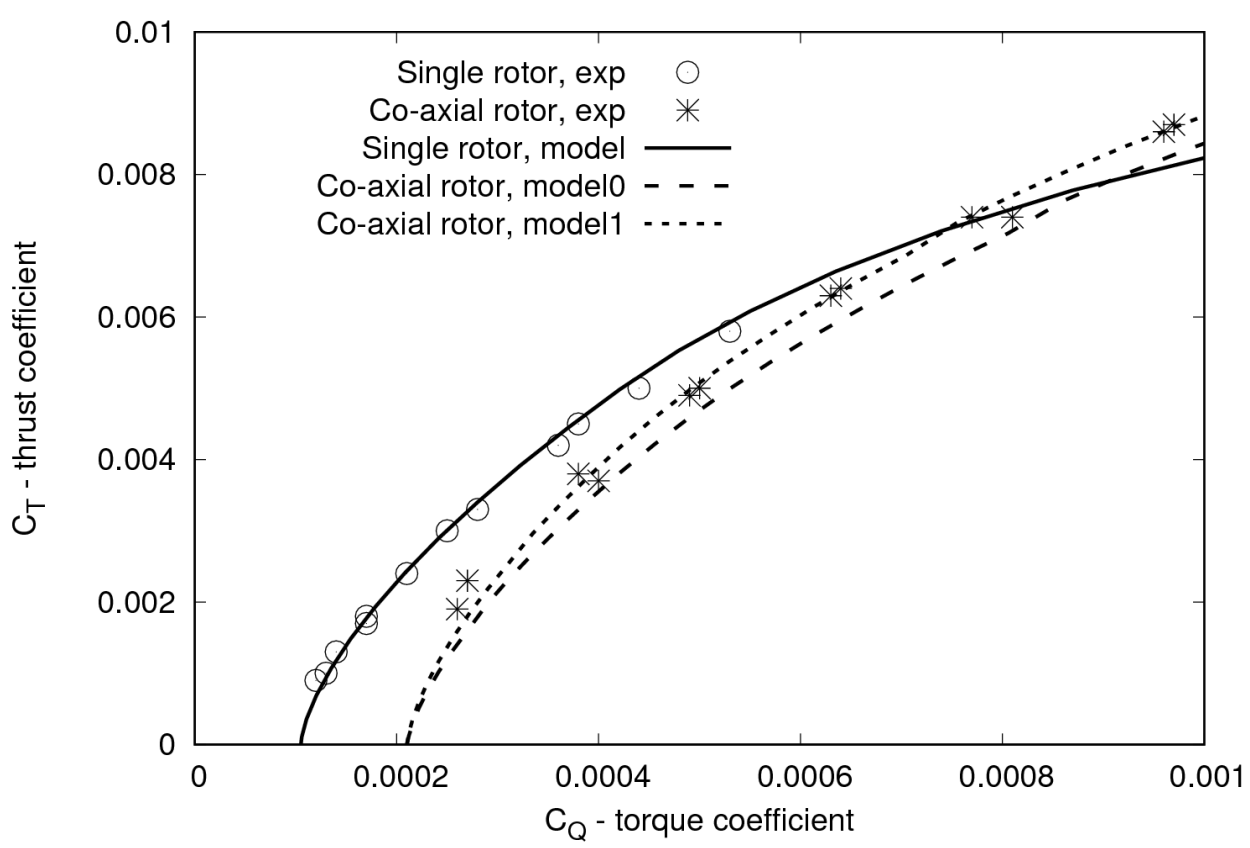

Figure 6: Variations of the thrust and torque coefficients of the Harrington two-blade rotors' staticthrust tests [21] and which are plotted for (a) Rotor 1 of the tapered chord and thickness blade and (b) Rotor 2 of the tapered thickness blade. Model 0 means $\mathrm{u}(\mathrm{x}, \mathrm{r})=\mathrm{u}(\mathrm{x}, \mathrm{r}=0)$ of Table 1 for $\mathrm{r}<\mathrm{R}$. Model 1 means $\mathrm{u}(\mathrm{x}, \mathrm{r})$ is found using Table 1. 


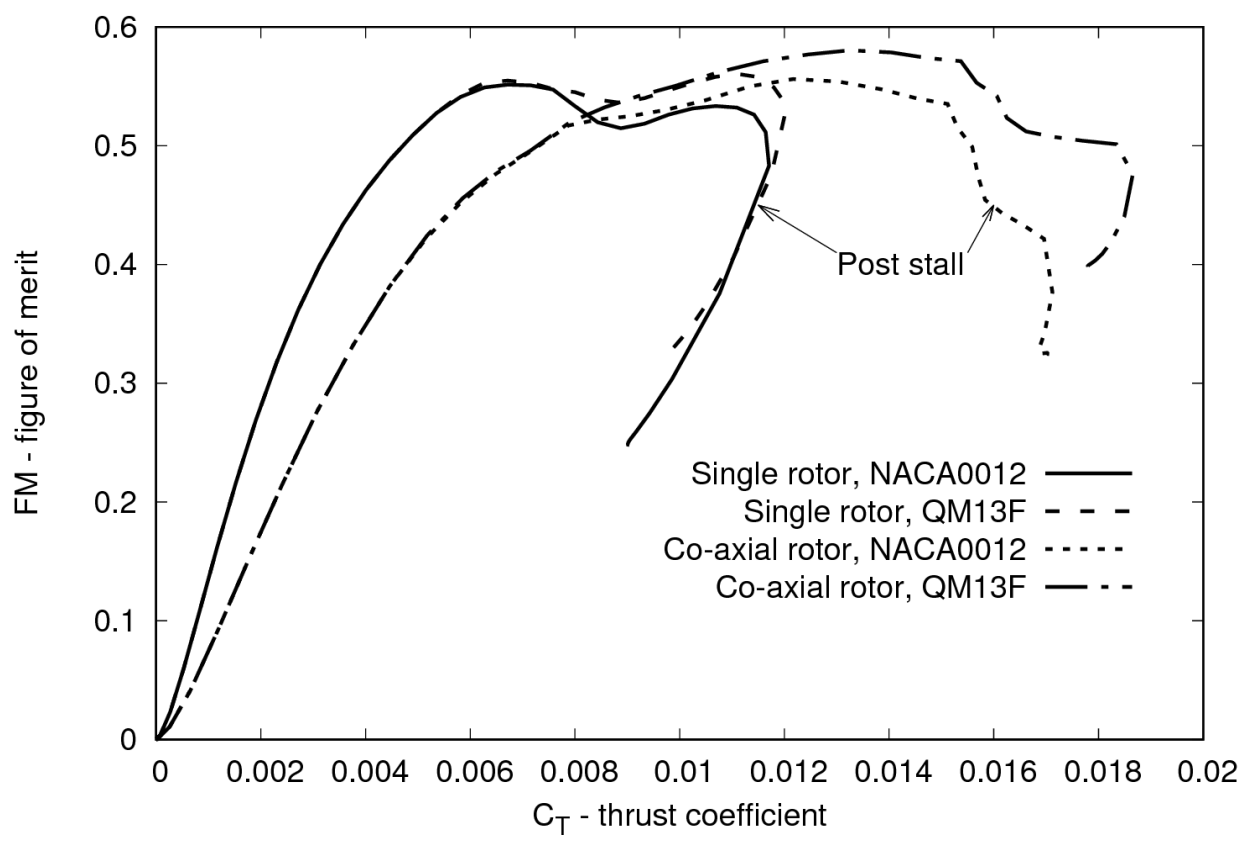

Figure 7: The variation of the figure of merit with the static thrust coefficient for a small re-scaled Harrington Rotor 2, where the blade profile is uniformly NACA0012 or QM13F of Fig 1a. 
(a)

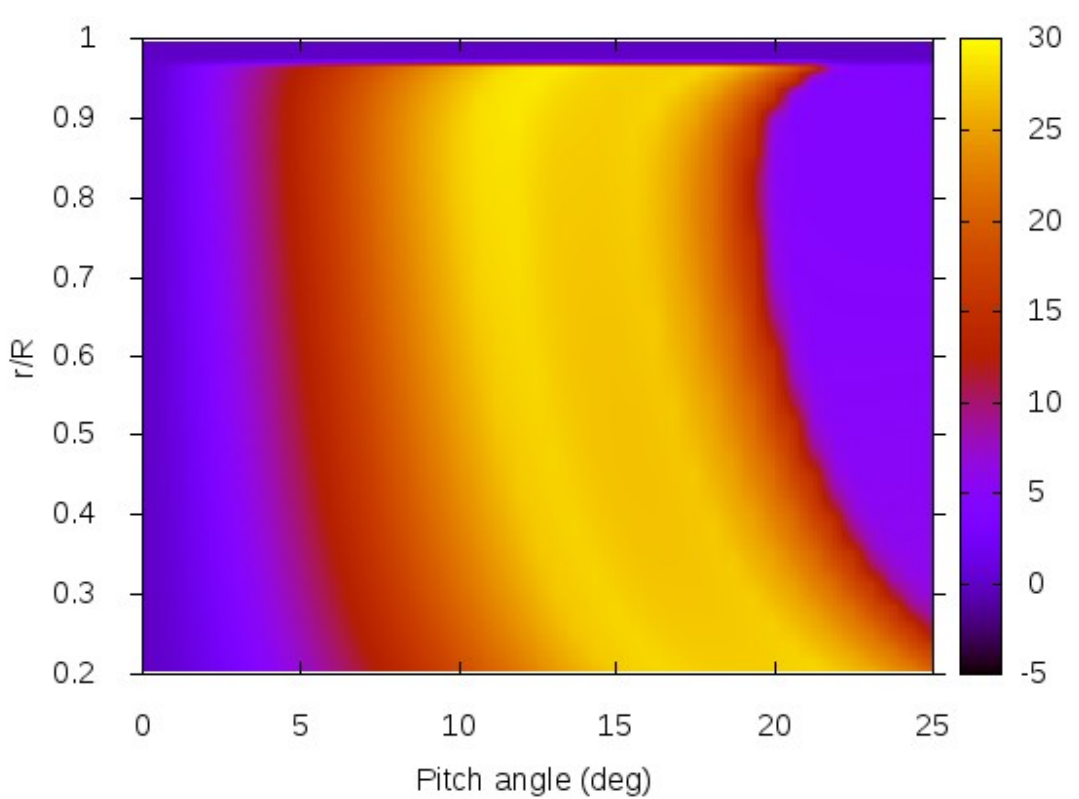

(b)

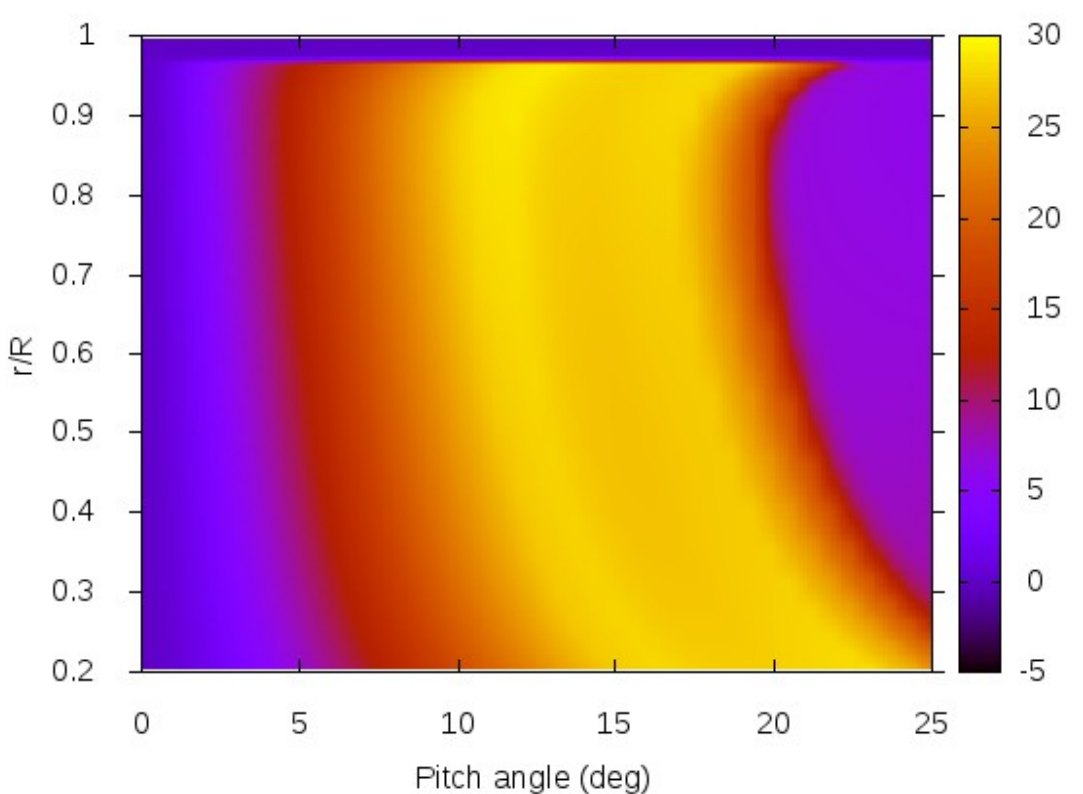


(c)

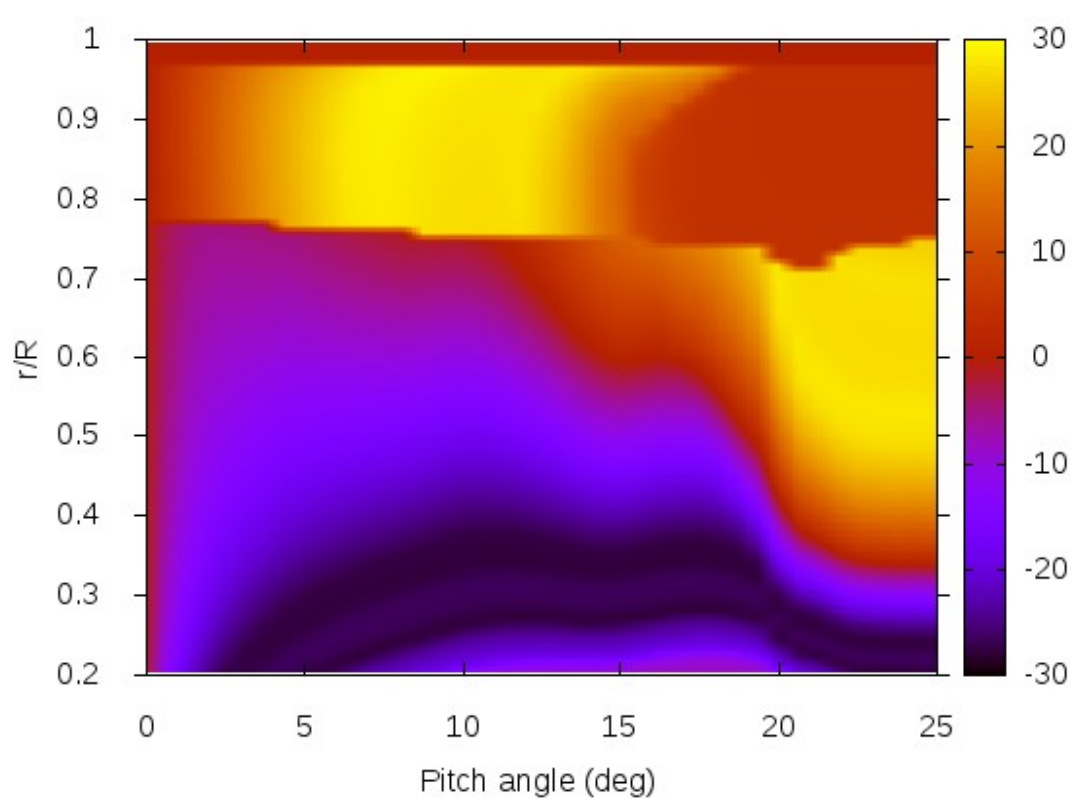

(d)

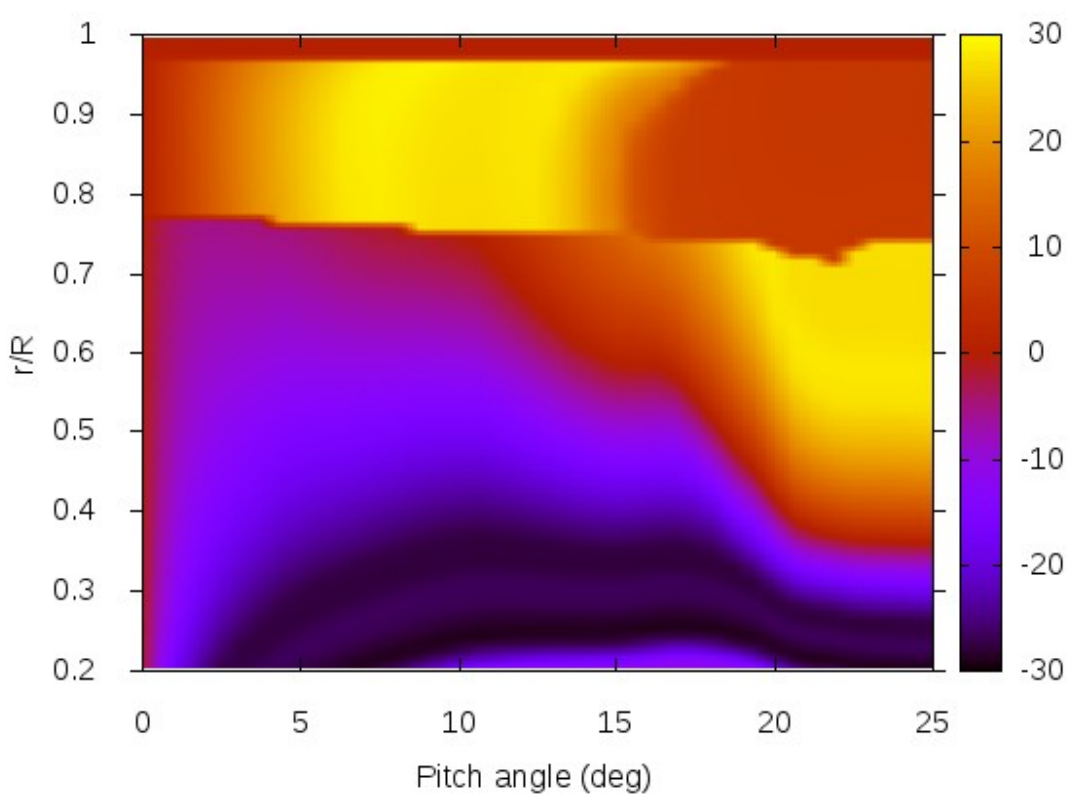

Figure 8: The spanwise variation of the blade profile's lift to drag ratio for the range of untwisted blade pitch angle $\beta$ corresponding to the FM plots of Fig 7 and which are plotted for (a) the upper rotor with the NACA0012 profile, (b) the upper rotor with the QM13F profile, (c) the lower rotor with the NACA0012 profile and (d) the lower rotor with the QM13F. 
(a)

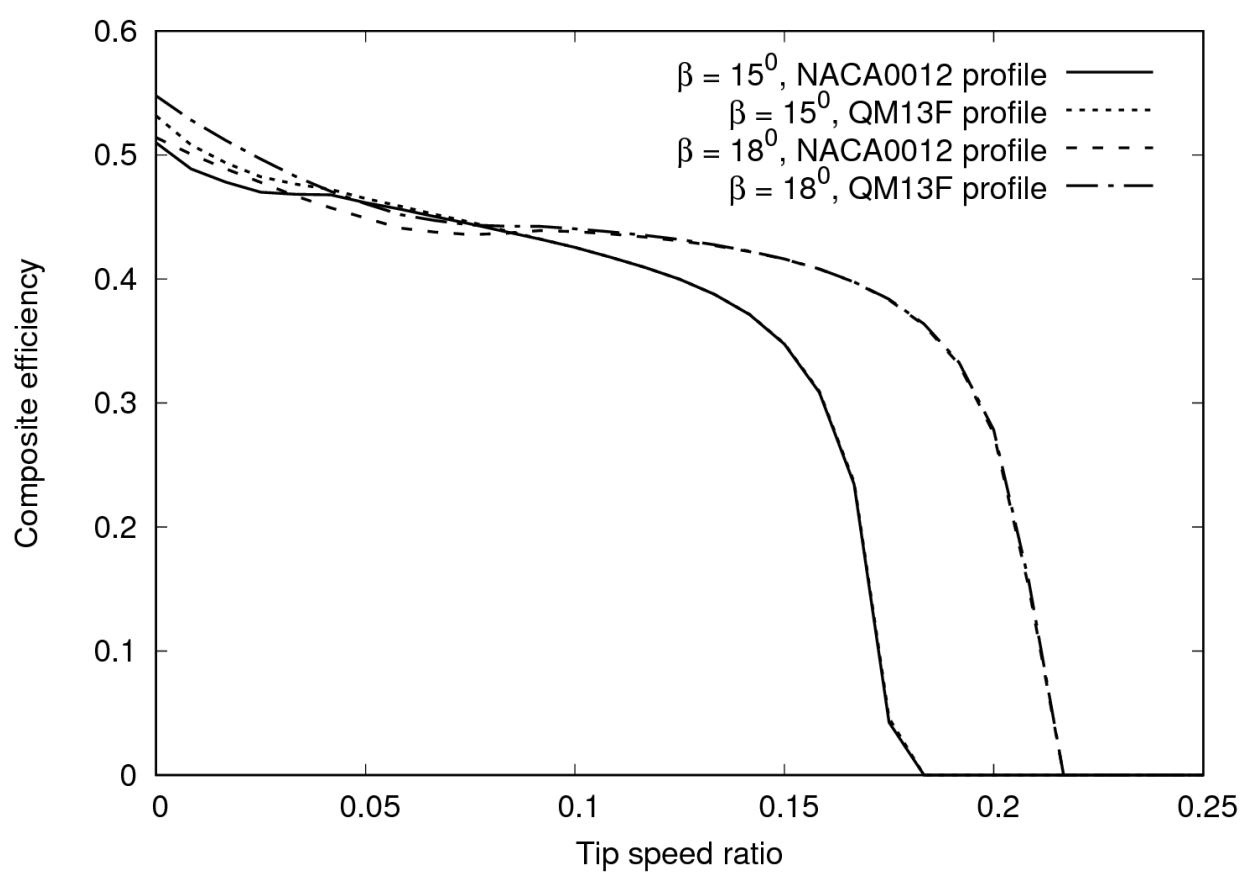

(b)

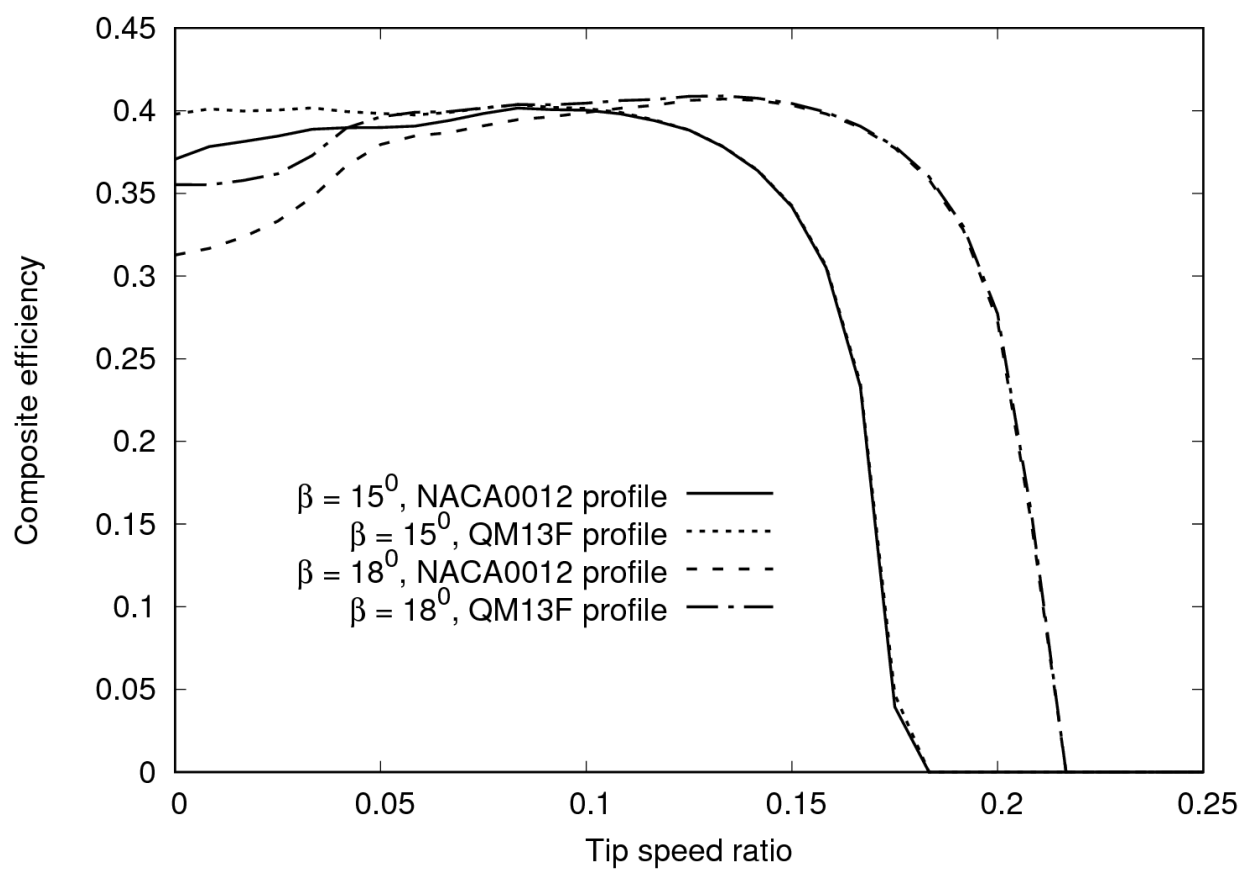

Figure 9: Variation of the composite efficiency $\eta_{\mathrm{C}}$ with the tip speed ratio $\lambda_{\infty}$ for the small rescaled (a) single and (b) coaxial Harrington Rotor 2, where both are at axial translation and $\beta$ is the untwisted blade pitch angle. 
(a)

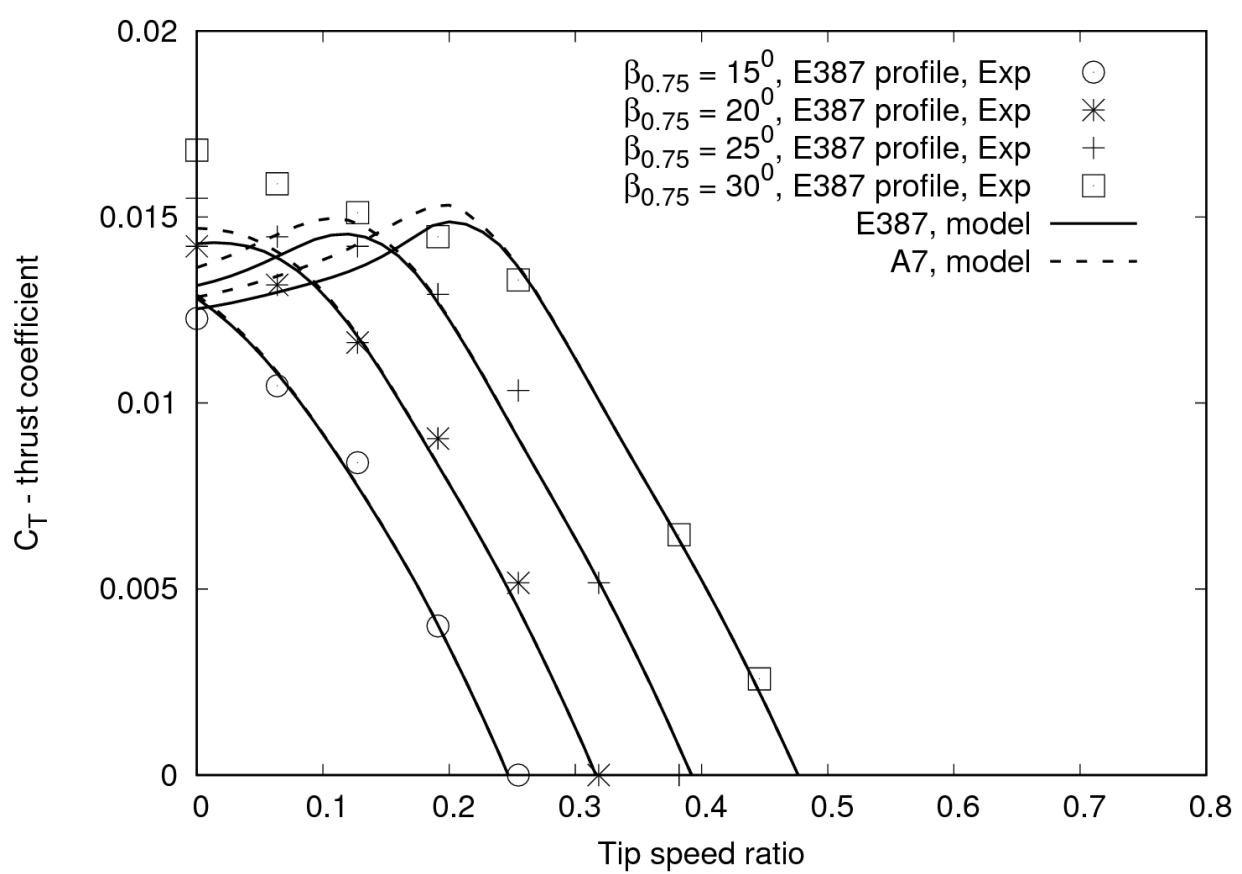

(b)

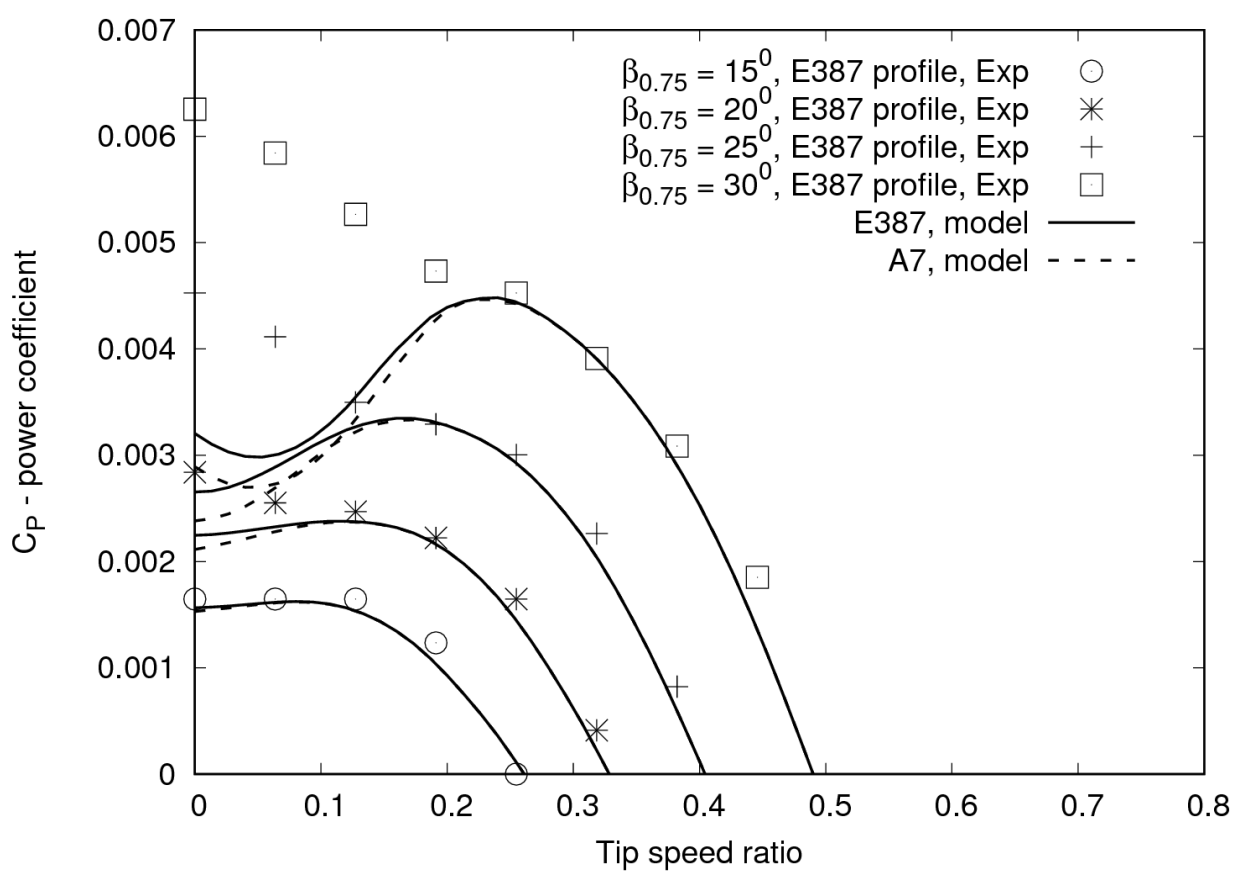

Figures 10: The variations of the (a) thrust and (b) power coefficients for the two-blade twisted COMP propeller having the E387 profile [23] and the redesigned A7 profile. 